Journal of Fluid Mechanics

http://journals.cambridge.org/FLM

Journal of

Additional services for Journal of Fluid Mechanics:

Email alerts: $\underline{\text { Click here }}$

Subscriptions: $\underline{\text { Click here }}$

Commercial reprints: $\underline{\text { Click here }}$

Terms of use : $\underline{\text { Click here }}$

\title{
One-to-few and one-to-many branching tube flows
}

\section{F. T. SMITH and M. A. JONES}

Journal of Fluid Mechanics / Volume 423 / November 2000, pp 1 - 31

DOI: 10.1017/S0022112000002019, Published online: 03 November 2000

Link to this article: http://journals.cambridge.org/abstract_S0022112000002019

How to cite this article:

F. T. SMITH and M. A. JONES (2000). One-to-few and one-to-many branching tube flows. Journal of Fluid Mechanics, 423 , pp 1-31 doi:10.1017/S0022112000002019

Request Permissions : $\underline{\text { Click here }}$ 


\title{
One-to-few and one-to-many branching tube flows
}

\author{
By F. T. SMITH AND M. A. JONES \\ Department of Mathematics, University College London, Gower St., London, WC1E 6BT, UK
}

(Received 21 May 1999 and in revised form 13 March 2000)

Branching tube flows are examined, for one mother to two, three or more daughter tubes. The case of many daughters (abrupt multi-branching) models blood flow through an arteriovenous malformation in the brain, while that of very few daughters (gradual branching) applies elsewhere in physiology and surgical grafting, as well as other applications including industrial ones. Theory and computation are presented for two- and three-dimensional motions, under the viscous and inviscid effects of small changes in mass flux between the daughter tubes, area expansion and turning of the flow. Specific configurations for which flow solutions are obtained are $(a)$ with two large daughters, $(b)$ with one small daughter/side branch, and $(c)$ with multiple small daughters.

The numerous physical mechanisms acting concern overall upstream influence and through-flow, and flow separation and criteria for its avoidance, as well as criteria for the amount of turning and area expansion possible without energy loss and other factors associated with separation, and the role of the branching geometry versus that of the mass-flux distribution in the daughters. In particular, configuration $(a)$ allows substantial separation-free turning and expansion only with certain shaping of the outer wall and an area expansion ratio typically less than 1.2 , whereas more daughters involve a balance between geometry and mass flux. In (b), an abrupt pressure jump is induced at the mouth of the small daughter, near which mass-flux effects tend to dominate over geometrical shaping effects. In $(c)$, as the number of daughters increases, the amount of separation-free turning and expansion is found to increase substantially, and the distributed mass-flux influence readily overrides the geometrical influence throughout the branching; there is also an integrated upstream effect of the multi-branching on the incident mother flow even though each daughter flow acts as if independent. Tentative designs based on wall shaping, flux distributions and divider placement are considered for flow improvement/surgery.

\section{Introduction}

This investigation concentrates on certain basic internal branching flows, the branchings being from one mother tube to few or many daughter tubes downstream. The fluid is assumed incompressible, and both inviscid and viscous effects are taken into account in the theory and computations.

The motivation for the work is primarily from physiological flows where branches are extremely common, throughout the human body for example, and involve various complex geometrical configurations and flow conditions associated with different ranges of Reynolds numbers, pulsatility, wall flexibility and so on. Many previous studies (for example Roach, Scott \& Ferguson 1972; Motomiya \& Karino 1984; 
Fisher \& Fieman 1990) address typical/modelled aortic or carotid branchings for instance with one mother flow bifurcating into only two or three daughters at most; see Lighthill's (1972) comments on early generation branches in the cardiovascular system and in the lungs. Our particular interest was also aroused by the clinical work of Mr N. D. Kitchen, at the Institute of Neurology, on arteriovenous malformations (AVMs) in the brain. An AVM constitutes a sudden division of a feeding artery into numerous thin vessels, as if for an abrupt multiple branching. These vessels overall appear to enlarge the flow area locally, serving as direct shunts for blood flow between the high-pressure arterial system and the low-pressure venous system. AVMs are associated with a large pressure gradient but small vascular resistance (Handa et al. 1993; Miyasaka et al. 1993; Young et al. 1994; Hademenos \& Massoud 1997 and references therein), yielding relatively rapid blood flow through the multi-branched system. AVMs represent a major life threat (stroke), particularly to people aged under 50. Several surgical procedures exist, such as clipping or glue casting, to attempt to reduce, and/or alter favourably the distribution of, the mass flux through the AVM and hence through the feeding artery upstream: Neil Kitchen, private communications, 1997-1999. These procedures suggest (as indeed did Neil Kitchen) a study on the effects of varying the mass-flux distributions across the daughter tubes in an abrupt multi-branching system and on the crucial impact of upstream influence, from the daughters to the mother tube flow. The same aspects hold for other branchings but they seem particularly significant in the multi-branching context. Again, models have been proposed for the intracranial blood vessel network (e.g. Hademenos, Massoud \& Viñuela 1996; Gao et al. 1997), incorporating an AVM model, and these also require improved understanding of the substantial influence from an AVM-like local multiple branching on the total contained flow. Most of the branched flows of interest here are predominantly laminar, with Reynolds numbers varying from a few hundred to a few thousand, and can be taken to be quasi-steady because of the short length scales near the branching and hence short typical time scales compared with the long-scale oncoming flow.

More appreciation of branched flows, in particular a prediction for the critical turning angle at which flow reversal/separation is encountered, should also point to improved designs for other surgical procedures, as in grafting (e.g. Lei, Kleinstreuer $\&$ Archie 1997), and in such non-physiological applications as plumbing, engine and machinery dynamics, for example in fluid ejectors and distributors. The consideration of efficient designs for the latter is close to that of the highly efficient flow through an AVM branching. Indeed another prime new feature of concern is that of multibranching, with say three or more daughter tubes, and the need for increased physical understanding of the overall fluid dynamical response and how it varies with the number of branches present (as well as with shaping and with mass-flux distribution).

Among the simplest two-dimensional and three-dimensional branched flow models, in theory, are those for one-to-two branchings posed and studied by Smith (1976a, 1977a), Bates (1978) and Brotherton-Ratcliffe (1987), for either fully developed incident motions or undeveloped entry motions in the mother tube. These are specifically for straight plane-channel or circular-pipe branches, with (thus) negligible turning of the outer walls and negligible area expansion of the contained flows, and with a single, essentially flat, divider wall between the two daughter tubes. Subsequent studies in the three-dimensional setting are by Bennett (1987) and by Walton \& Smith (1997) who find pronounced longitudinal-vortex behaviour close to the intersection between the divider and the outer wall.

The papers mentioned just above point to the critical angles or relative thicknesses 
of the branching geometry being of the order of the inverse Reynolds number to the one-third power if, as here, the focus is on distances (measured from the branching) not much greater or less than the characteristic cross-sectional dimension $\left(a_{D}\right)$ of the tube and the Reynolds number $(R)$ is large. The tube is, for example, either a channel with width $2 a_{D}$ or a pipe of radius $a_{D}$, while the typical $R$ values of practical interest are about 300-3000, giving critical $R^{-1 / 3}$ values, about 0.1 , which are suitably small for the theory. The latter values are close to the value, about 0.2 , noted by Lighthill (1972) for early generations of branching in which he considers the area expansion ratio $\beta$ (of the combined area of the daughters to the area of the mother tube) at a branch and quotes 1.2 as the typical maximum value for avoidance of flow separation.

Likewise, approximations for large $R$ are found to work well at practical finite $R$ values in internal flows according to comparisons in Dennis \& Smith (1980), Smith $(1977 b, 1980)$ and Sobey $(1980)$, for example, and serve as a reasonable model for providing physical insight. Direct numerical simulations have also been performed for branchings, see for instance Hademenos \& Massoud (1997), shedding some light on one-to-two branching flows, although much less appears to have been done on the more difficult case of one-to-many branches.

The present investigation starts with two-dimensional channel flow, and tackles certain basic issues of this branching first. Many of these then apply immediately in the three-dimensional setting addressed later. In a similar vein, quasi-steadiness is assumed (see also earlier comments) although we may expect fully unsteady motions to respond similarly over the wide range in which they do not disrupt the flow scales involved in the branchings. The mother tube and its fluid flow are supposed to be straight upstream although the local theory can accommodate non-straight input instead, just as it accommodates non-straight daughter tubes and flows downstream. The fluid is taken as Newtonian, and the walls are taken to be fixed (see also Luo \& Pedley 1996, 1998). The main questions arising, which need to be answered first for basic cases as considered here, include that of whether flow separation can be avoided at a branching, whether the cross-sectional area can expand without inducing separation, how much upstream influence is provoked by a one-to-few or a oneto-many branching, and what is the role of mass-flux variations in the daughters, especially in multi-branchings?

Several physical mechanisms are found to be present, some well known, some not, in these branching motions. In particular there is in the present setting a fairly clear distinction between, on the one hand, the flow properties in mid-flow branchings corresponding to large daughter tubes, where the branched geometry plays an important role, and, on the other, those in near-wall branchings of a small daughter or side branch and in multiple branchings with many small daughters, where the role of the daughters' mass flux usually overwhelms that of the branching geometry. Further, as the number of daughter branches increases, increased turning and area expansion are found possible with separation-free flow (a good design property for grafting, in principle, although subject to increased viscous resistance for thinner tubes or longer streamwise scales), while the mother flow ahead of the branch is affected mostly by the daughter mass-flux distribution.

Here we assume usually that small changes in the mass fluxes are prescribed, rather than pressures in the daughter tubes as well as in the mother, and the distribution of the pressure head loss can then be predicted for given total mass flux and hence Reynolds number. These fluxes are set by control of the far downstream pressures in each branch relative to the upstream pressure in many contexts, but the main pressure control is not usually on the streamwise length scale $a_{D}$ of current interest. Instead it 
is on the larger viscous scale of order $a_{D} R$, mostly beyond the daughter mouths, and this larger-scale flow helps to fix the mass flux for each daughter of the branching. Such viewing of the imposed pressure drops over a relatively long streamwise scale is familiar for most flows through slender tubes and, in the present branching flows, it leaves the pressure drops as not necessarily known over shorter streamwise scales near the branching itself. Further, in other contexts such as the surgical procedures for AVMs the flow control is based not on direct pressure adjustments anyway but more on adjusting the geometry or flux, or both, e.g. as in the glueing or clipping of daughter tubes. So although a formulation with prescribed pressure drops could be applied in all such cases a formulation with prescribed fluxes is just as relevant, if not more so, over the current short length scale. Further properties are described in recent work by Smith (2000a). An exception arises for many small daughters, where the viscous length scale shortens and so the two formulations come together, in which case both should be considered.

Since in these branched flows the incident motion must adjust itself significantly upstream of the branching, in order to provide the correct mass flux for each daughter, we consider streamwise distances comparable with $a_{D}$, prior to any largerscale fully viscous development further downstream. This is as anticipated above. Upstream influence is indeed a key feature throughout and is associated mostly with distances comparable with $a_{D}$. The influences of wall shapes and the daughters' mass fluxes are also examined below with a view, however remote, to possible designs for reconstructive surgery for example.

Clearly the aim in this work is to develop a simple model first. Some of its important limitations have been mentioned already, in the last but three paragraph. In addition viscous effects are insignificant on the current length scales except near the outer wall, and the whole assumed geometry is idealized. The applicability of this simple model remains to be seen. Although direct application to the practical settings described earlier is unlikely, the comparisons at finite $R$, also noted earlier, are encouraging and the theory is expected to establish guidelines, scales and the relevant physical mechanisms, as well as indicating future studies, for the branching flows in question.

Section 2 describes the branching flow structure, which is the same essentially for two- or three-dimensional cases, with a linear inviscid core supplemented by a nonlinear viscous outer wall layer. The core motion is unaffected by the outer wall geometry in the present setting. Attention is then paid first to symmetric planar branched motions, leaving aside the special extra features of non-symmetric planar motions in Smith (1977b), Smith \& Duck (1980), Borgas \& Pedley (1990) and others. Three main configurations are studied in $\S \S 3-5$ for planar motions. Thus $\S 3$ considers one-to-two branching with two large daughters, corresponding to a central divider downstream. The effects of various incident velocity profiles are examined, including those of plug flow, uniform shear flow and fully developed flow, here and in subsequent sections. In $\S 4$ a small daughter is considered, giving a side branch. This can produce, near the mouth of the daughter tubes, a rapid acceleration or deceleration and an abrupt pressure jump; an inkling of the latter is given in some of the flow computations by Luo \& Pedley mentioned above for flexible walls, while other flows inducing related pressure jumps are discussed by Jones \& Smith (2000), Jones (2000), Bowles \& Smith (2000) and Smith (2000b). Section 5 describes one-to-many branching flows, where again there is an abrupt pressure jump. Such multi-branching motions force the mass-flux or pressure requirement of the multiple daughter system to apply immediately at the branching section, in controlling all the mother flow upstream, whereas all the daughter flows act independently beyond the daughter 
mouths. Three-dimensional branchings are then addressed in $\S 6$, concentrating on one-to-two and (more especially) one-to-many branches of a mother pipe of circular cross-section. The same properties hold as for the planar cases except for the addition of corner vortices as in Walton \& Smith (1997). Section 7 provides further comments.

\section{The general flow structure in two dimensions}

The Cartesian coordinates $x, y, z$, corresponding velocity components $u, v, w$ and the pressure variation $p$ used here are non-dimensionalized with respect to a typical cross-sectional width $\left(a_{D}\right)$ and incident velocity $u_{D}$, so that in the planar cases of $\S \S 2-5$ the channel half-width is unity and the incident velocity profile $u=u_{0}(y)$ in the streamwise direction $x$ is of order 1 . The Reynolds number $R \equiv u_{D} a_{D} / v_{D}$ is large, $v_{D}$ being the kinematic viscosity of the incompressible fluid. For a straight mother channel the profile upstream $u_{0}(y)$ is taken to be positive except at the undisturbed channel walls $y=0,2$ where it is zero, with an order 1 derivative, $u_{0}(y)$ is symmetric about the centreline $y=1$, and flow symmetry about $y=1$ is assumed throughout. The stream function is $\psi$ for the planar case, with $\psi$ zero at the wall $y=0$, the threedimensional case being addressed later in $\S 6$. Branching starts at the station $x=0$ say, from the single mother to an order-one number $N$ of daughters downstream.

A small expansion of the characteristic cross-sectional area enclosed and small directional changes are examined first below, followed subsequently (see §3.3) by consideration of larger turnings due to the tube branching. The streamwise length scale $|x|$ of interest is of order unity.

For large $R$, if the typical gap width $\alpha$ of a daughter tube is $O(1)$, then its typical wall (divider) thickness is taken to be of size $O\left(R^{-1 / 3}\right)$. The size follows from the order of magnitude $|y|$ of the viscous layer thickness at the outer wall and its inertialviscous balance $u \partial / \partial x \sim R^{-1} \partial^{2} / \partial y^{2}$ giving $|y| / 1 \sim R^{-1} /|y|^{2}$ and so $|y| \sim R^{-1 / 3}$ there (as anticipated in $\S 1$ ). This outer-wall thickness is strictly much greater than the $O\left(R^{-1 / 2}\right)$ thickness of each viscous Blasius boundary layer that is produced on each inner divider, and hence the outer wall layer is exceptional as it is the most sensitive to changes taking place in the inviscid core flow during the tube branching. See figure 1. The particular scale $R^{-1 / 3}$ of divider thickness makes these changes affect the motion in the outer wall layer in a nonlinear manner, thus raising the possibility of flow separation occurring there.

\subsection{The core}

The divider shape has $y=y_{n}+R^{-1 / 3} f_{n}^{ \pm}(x)$ say, on the typical $n$th divider wall for $x>0$, with $\left|f_{n}\right| \sim 1, f_{n}(0)=0$ if the divider's leading edge is not too blunt, and $n$ runs from 1 to $N$. So the streamwise velocity $u$ in the core where $0<y<1$ has the form $u_{0}(y)+R^{-1 / 3} \tilde{u}(x, y)$ and similarly for $\psi, p$, and the Navier-Stokes equations then reduce to the inviscid small-perturbation system

$$
u_{0} \nabla^{2} \tilde{\psi}=u_{0}^{\prime \prime} \tilde{\psi}
$$

for $\tilde{\psi}(x, y)$ where $\tilde{u}=\partial \tilde{\psi} / \partial y$ and $\nabla^{2}$ denotes $\left(\partial^{2} / \partial x^{2}+\partial^{2} / \partial y^{2}\right)$. The boundary conditions are

$$
\begin{aligned}
& \tilde{\psi}=-u_{0}\left(y_{n}\right) f_{n}^{ \pm}(x)+c_{n} \quad \text { at } \quad y=y_{n} \pm, \\
& \tilde{\psi} \rightarrow 0 \quad \text { far upstream, } \\
& \tilde{\psi}=0 \quad \text { at } \quad y=0 \quad(\text { all } x), \quad y=1 \quad(\text { for } x<0),
\end{aligned}
$$




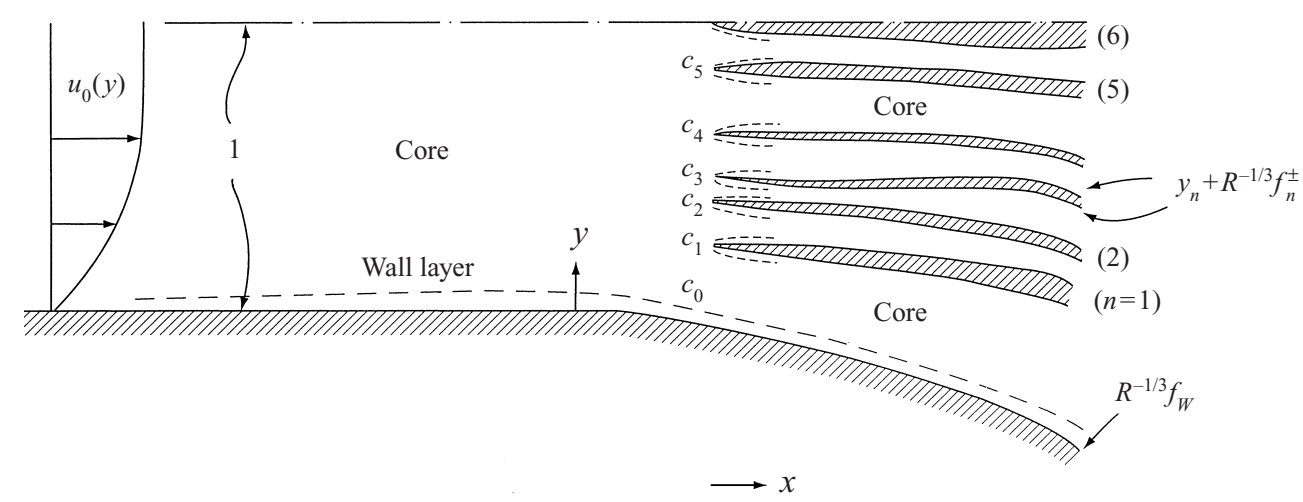

FIGURE 1. Sketch of the multi-branching flow structure, showing the inviscid core in the mother and daughter tubes, the $R^{-1 / 3}$ viscous outer wall layer and the $R^{-1 / 2}$ viscous layers on the dividers between daughter tubes, in non-dimensional quantities. The scaled mass-flux values are $c_{0}$ to $c_{N}$ with $N=6$ in this case.

along with regularity of $\tilde{\psi}$ within the core motion. Here $(2.1 b)$ is for tangential flow on each divider, sited at $y=y_{n}+R^{-1 / 3} f_{n}^{ \pm}$with the characteristic $y_{n+1}-y_{n}$ gap being $\alpha$, of order 1 , and $n=1,2, \ldots, N$, with $y_{N}=1$. The given constants $c_{n}$ are associated with the small changes $c_{n+1}-c_{n}$ in mass flux prescribed for each daughter channel as in figure 1 , along with $c_{0}=c_{N}=0$ for overall mass conservation, in line with the comments on flow control in $\S 1$.

The constraint $(2.1 c)$ is to satisfy the incident conditions, whereas $(2.1 d)$ is to yield tangential motion at the outer wall and symmetry along the centreline. We notice that there is no influence yet from the outer-wall shape $y=R^{-1 / 3} f_{W}(x)$. The coefficient multiplying $f_{n}^{ \pm}(x)$ in $(2.1 b)$ confirms the exceptional nature of the outer-wall flow since there $u_{0}$ is zero, while on the dividers the fact that the Blasius boundary-layer thickness is proportional to $R^{-1 / 2}\left[u_{0}\left(y_{n}\right)\right]^{-1 / 2}$ and so increases as $u_{0}$ decreases also anticipates the outer wall-layer thickness being larger than the thicknesses of the divider boundary layers, so that all of the latter can be neglected, at least in the current setting.

The core flow response above can also be expressed in terms of a pressure equation for example, with $\tilde{\psi}$ replaced by $\tilde{p}$ and $u_{0}^{\prime \prime}$ by $2 u_{0}^{\prime}$ in $(2.1 a)$, but we shall keep mostly to the mass-flux formulation. The inviscid core problem $(2.1 a-d)$ is a closed one, determining among other properties a slip-velocity effect $u_{W}(x) \equiv \tilde{u}(x, 0)$ at the outer wall.

\subsection{The wall-layer flow}

The outer wall layer has $y=R^{-1 / 3}\left(Y+f_{W}(x)\right)$, with $Y \sim 1$ incorporating a Prandtl shift, and the flow is driven partly by the slip effect $u_{W}(x)$. Thus now $u, \psi, p$ are respectively $R^{-1 / 3} U, R^{-2 / 3} \Psi, R^{-2 / 3} P(x)$ to leading order, giving the boundary-layer equations

$$
U=\frac{\partial \Psi}{\partial Y}, \quad U \frac{\partial U}{\partial x}-\frac{\partial \Psi}{\partial x} \frac{\partial U}{\partial Y}=-P^{\prime}(x)+\frac{\partial^{2} U}{\partial Y^{2}}
$$

with $\partial P / \partial Y$ zero from the $y$-momentum balance, and with the boundary conditions of no slip at the outer wall, of matching with the core flow solution and of matching upstream, so that

$$
U=\Psi=0 \quad \text { at } \quad Y=0
$$




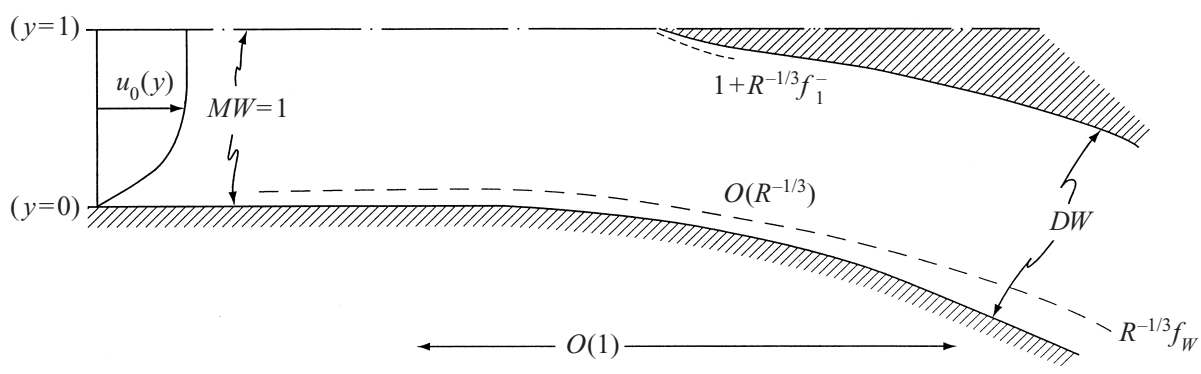

FIGURE 2. Two large daughter tubes, with symmetry about $y=1: \S 3$. Here $N=1$, the mother half-width and daughter width are $M W, D W$ respectively, and the wall shapes (divider, outer, in turn) are given by $f_{1}^{-}(x), f_{W}(x)$ with $x$ of order unity.

$$
\begin{gathered}
U \sim \lambda[Y+B(x)] \quad \text { as } \quad Y \rightarrow \infty, \quad B \equiv \lambda^{-1} u_{W}(x)+f_{W}(x), \\
(U, \Psi, P) \rightarrow\left(\lambda Y, \frac{1}{2} \lambda Y^{2}, 0\right) \quad \text { as } \quad x \rightarrow-\infty,
\end{gathered}
$$

in turn. Here $\lambda$, denoting $u_{0}^{\prime}(0)$, is of order 1 . A normalization of $U, P, Y, B$ with $\lambda^{m}, m=2 / 3,4 / 3,-1 / 3,-1 / 3$ in turn, and rescaling, allows $\lambda$ to be replaced by unity in effect above; it is also found below that $u_{W}$ tends to scale with $\lambda$ anyway.

The influence of the outer-wall shape is now present explicitly in $(2.2 c)$, while that of the inner dividers and mass fluxes is felt via the slip velocity $u_{W}(x)$. This slip now acts as part of the effective displacement $B(x)$ because, with the incident linear profile $U$ being proportional to $Y$, an alteration in $U$ due to slip transforms directly into a displacement in $Y$. The conversion from slip to displacement is from Smith (1978) and related papers and it is important because it leaves the wall pressure unknown and thereby renders any flow reversal (separation) within the wall layer a regular process, despite the classical attached nature of the core flow. The influence of the inner dividers on the motion near the outer wall is therefore due to a pressurefeedback mechanism through the core, the ellipticity of which provokes the major upstream influence, in particular within the outer wall layer even though the latter of course contains no dividers itself. In general $u_{W}$ is non-zero in $x<0$ despite the branching being confined to $x \geqslant 0$. For the present context the effective displacement driving the viscous outer wall layer is dominated, ahead of the outer-wall turning, by the influences of the inner divider shapes and mass fluxes, and thereafter by a combination of those influences and the outer turning.

The branching flow properties hinge on solving the core problem $(2.1 a-d)$, to find $u_{W}(x)$ among other quantities, and then the wall-layer problem $(2.2 a-d)$, to find for instance the unknown scaled wall pressure $P(x)$ and the induced wall shear $\tau(x)[\equiv \partial u / \partial Y(Y=0)]$. The core problem is tractable for general $N$ in principle, for example by use of a Wiener-Hopf technique, but three specific cases are more helpful. These are studied in $\S \S 3-5$, followed by three-dimensionality in $\S 6$.

\section{With two large daughters}

In this fundamental branching the gap width $\alpha=1$, corresponding to the case $N=1$ (figure 2). The flow solution shows explicitly the effects of the single, central, divider wall and of the outer-wall shape. We continue first with slight turnings present, addressing substantial turnings afterwards. 


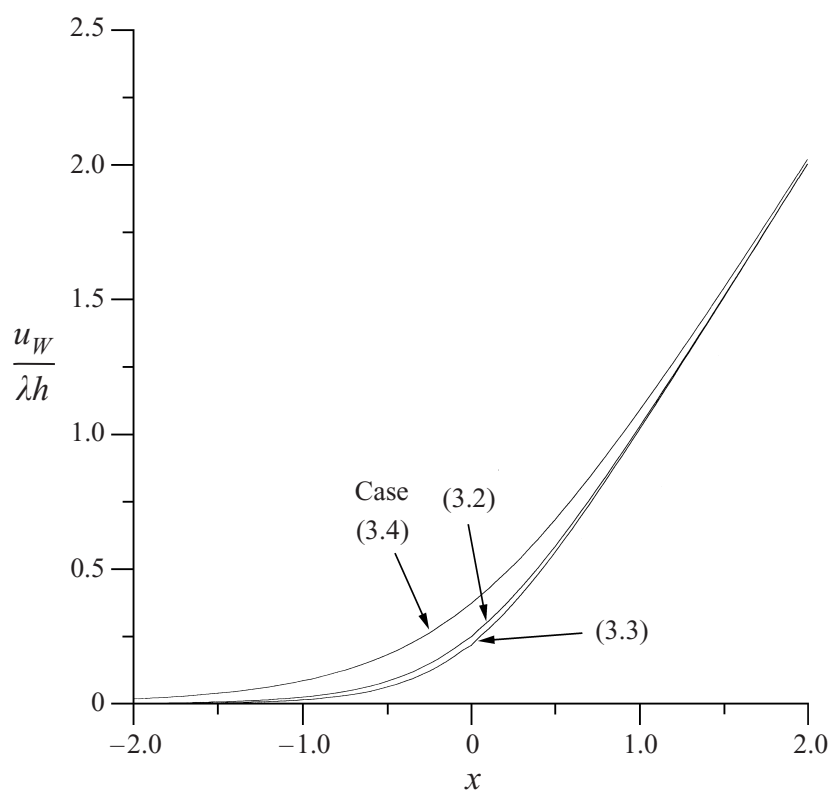

FIGURE 3. Slip-velocity results for two large daughter tubes calculated from the inviscid core, for the three incident profiles of plane Poiseuille flow, uniform shear and uniform velocity yielding (3.2), (3.3), (3.4) in turn, with a wedged divider shape.

\subsection{The core}

In the core the only $c_{n}$ values now are $c_{0}, c_{N}$, both zero, and $(2.1 b)$ becomes $\tilde{\psi}=$ $-u_{0}(1) f_{1}^{-}(x)$ at $y=1$.

For plane Poiseuille flow upstream, $u_{0} \equiv y-y^{2} / 2$, so that $\lambda=1, u_{0}(1)=1 / 2$, and the solution of $(2.1 a-d)$ can be obtained in series form (Tillett 1968)

$$
\tilde{\psi}=\left\{\begin{array}{l}
\sum_{1}^{\infty} \sigma_{n} \mathrm{e}^{\beta_{n} x} S_{n}(y) \text { for } x<0, \\
h x u_{0}(y)+\sum_{1}^{\infty} \tilde{\sigma}_{n} \mathrm{e}^{-\beta_{n} x} S_{n}(y) \text { for } x>0 .
\end{array}\right.
$$

Here the example chosen is that of a linearly increasing divider $f_{1}^{-}(x) \equiv-h x$ with $h$ a positive constant, $x \geqslant 0$, giving a wedge shape. The positive increasing eigenvalues $\beta_{n}$ and eigenfunctions $s_{n}(y)$, normalized such that $s_{n}^{\prime}(0)=1$, are as in Tillett's analysis in effect (although the $A_{n}$ values quoted in his table 2 and page 282 are incorrect); we find numerically the first five eigenvalues $\beta_{1}$ to $\beta_{5}$ to be respectively $2.58915,5.97050,9.19787,12.38573,15.55684$, with the corresponding coefficients $\sigma_{1}$ to $\sigma_{5}$ being $h$ times $0.31459,-0.10032,0.060612,-0.043617,0.034122$, respectively. These coefficients are found by equating $\tilde{\psi}, \partial \tilde{\psi} / \partial x$ at $x=0 \pm$ from $(3.1 a, b)$, which requires $\tilde{\sigma}_{n}=\sigma_{n}$ and $\Sigma \beta_{n} \sigma_{n} s_{n}=h u_{0} / 2$, and then applying orthogonality. From this we deduce

$$
u_{W}(x)=\sum_{1}^{\infty} \sigma_{n} \mathrm{e}^{\beta_{n} x} \quad(x<0), \quad \lambda h x+\sum_{1}^{\infty} \sigma_{n} \mathrm{e}^{-\beta_{n} x} \quad(x>0)
$$

for the slip velocity (figure 3).

Alternatively, for an input profile which is uniform in the middle, $u_{0}=1$ for 
$\delta \leqslant y \leqslant 1$, and linear next to that, $u_{0}=y / \delta$ for $0 \leqslant y \leqslant \delta$, with $\delta$ constant, the core flow is governed by Laplace's equation from (2.1a), with suitable continuity conditions across $y=\delta$. The solution when $\delta$ is near unity, corresponding to constant incident shear $u_{0}=y$ almost everywhere, stems directly from applying the constraints $(2.1 b-d)$ and gives

$$
\begin{aligned}
& u_{W}(x)=\pi \sum_{m=1}^{\infty} m(-1)^{m-1} f^{*}(-\mathrm{i} m \pi) \exp (m \pi x) \quad(\text { for } x<0), \\
& u_{W}(x)=\pi \sum_{n=1}^{\infty} n(-1)^{n-1} f^{*}(\mathrm{i} n \pi) \exp (-n \pi x)+E C \quad(\text { for } x>0)
\end{aligned}
$$

(figure 3), where $f^{*}$ denotes the Fourier transform in $x$ of $-f_{1}^{-}(x)$. The extra contribution $E C$ is a pole or branch-cut contribution from $f^{*}$ itself, and depends more on the specific divider shape, given that the transform of $u_{W}$ is equal to $\omega f^{*} / \sinh \omega$. An example is given shortly below. When $\delta$ is small, on the other hand, the main middle portion must satisfy $\partial \tilde{\psi} / \partial y \rightarrow 0$ as $y \rightarrow 0+$ instead of $(2.1 d)$. This is because the side portion has $\tilde{y}=y / \delta$ of $O(1)$, yielding $\tilde{u}_{0} \partial^{2} \tilde{\psi} / \partial \tilde{y}^{2}=\tilde{\psi} d^{2} \tilde{u}_{0} / d \tilde{y}^{2}$ to leading order from $(2.1 a)$ (generalizing to $u_{0} \approx \tilde{u}_{0}(y / \delta)$ here), so that $\tilde{\psi}=\tilde{a} \tilde{u}_{0}\left(1+O\left(\delta^{2}\right)\right)$ to satisfy $(2.1 d)$ (which gives $u_{W} \sim \delta^{-1} \tilde{a} \mathrm{~d} \tilde{u}_{0} / \mathrm{d} \tilde{y}(\tilde{y}=0)$ ) with $\tilde{a}(x)$ to be determined, and hence $\tilde{\psi} \rightarrow \tilde{a}, \partial \tilde{\psi} / \partial \tilde{y} \rightarrow 0$ at large $\tilde{y}$, indicating the above requirement for the middle portion. It follows then that the middle section yields formally (see figure 3 )

$$
\begin{gathered}
\frac{u_{W}(x)}{\lambda}=\sum_{m=1}^{\infty}(-1)^{m-1} f^{*}\left[-\mathrm{i}\left(m-\frac{1}{2}\right) \pi\right] \exp \left\{\left(m-\frac{1}{2}\right) \pi x\right\} \quad(x<0), \\
\frac{u_{W}(x)}{\lambda}=\sum_{n=1}^{\infty}(-1)^{n-1} f^{*}\left[\mathrm{i}\left(n-\frac{1}{2}\right) \pi\right] \exp \left\{-\left(n-\frac{1}{2}\right) \pi x\right\}+E C \quad(x>0) .
\end{gathered}
$$

The wall-shear factor $\lambda=\delta^{-1} \mathrm{~d} \tilde{u}_{0} / \mathrm{d} \tilde{y}(\tilde{y}=0)$ is of course large here, and we notice that the wall slip velocity scales with $\lambda$, this representing a general trend. In the case $f_{1}^{-}(x)=-h x \exp (-\epsilon x)$ for $x \geqslant 0$, with $h, \epsilon$ being positive constants, $E C$ in $(3.4 b)$ is $h \exp (-\epsilon x)(x-\tan \epsilon) \sec \epsilon$ (and similarly in $(3.3 b))$. This becomes $h x$ in the limit as $\epsilon$ tends to zero, corresponding to a wedge-shaped divider, as in $(3.1 a, b)$, and then $u_{W} \sim \lambda h x$ increases linearly far downstream. The latter result generalizes, for a shape $f_{1}^{-}(x) \sim-h\left(\gamma_{0}+\gamma_{1} x\right)$ at large positive $x$, with $\gamma_{0}, \gamma_{1}$ constants, to give accelerating near-wall flow with

$$
u_{W} \sim \lambda h\left(\gamma_{0}+\gamma_{1} x\right) \quad \text { as } \quad x \rightarrow \infty
$$

for a general $u_{0}(y)$ profile, in agreement with (3.2) in particular.

For all the incident flows above, the induced slip velocity is exponential, and positive, in $x$ sufficiently upstream of the branching. That seems a general property, in addition to (3.5) downstream. Moreover, the closeness of the $u_{W} /(\lambda h)$ variations with $x$ for the three cases (3.2)-(3.4) is striking, in figure 3, for three such different incident flows.

\subsection{The wall-layer properties}

Computations for the resulting viscous wall-layer flows were performed with an implicit box method, e.g. see Rothmayer \& Smith (1998), and results are presented in figure 4 under various conditions, in Poiseuille flow. Figure $4(a)$ has two cases (denoted 1,2), each with a straight divider (dvdr) and wall shape (gsh) that produce 
10
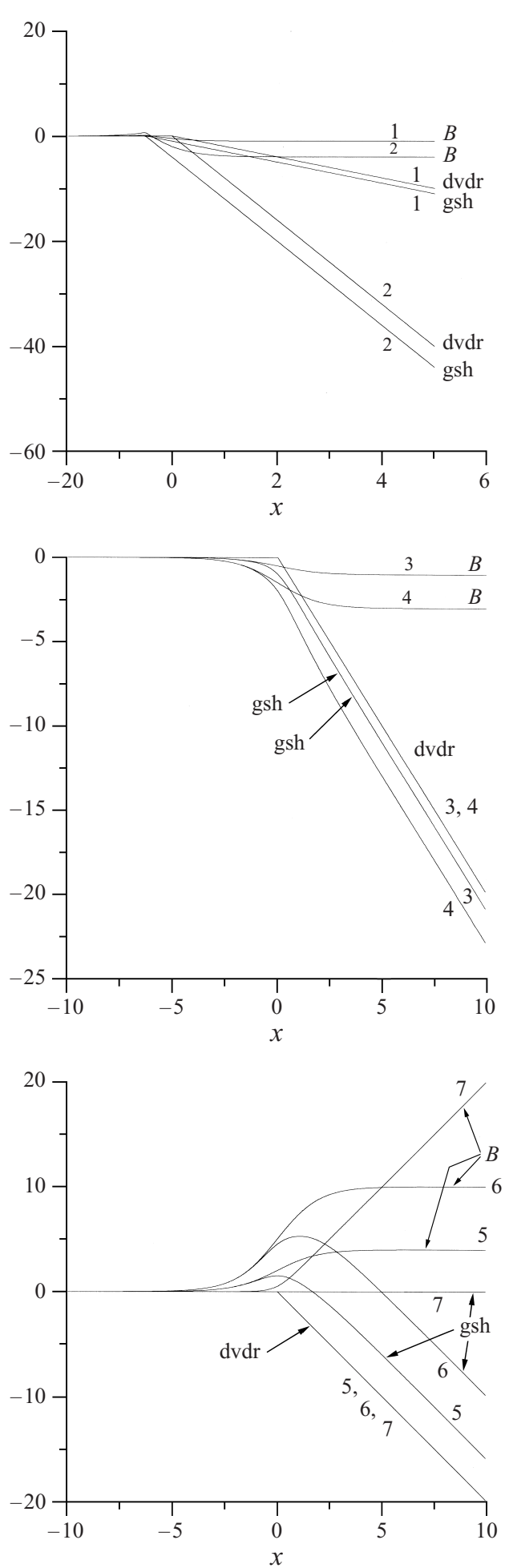

(a)

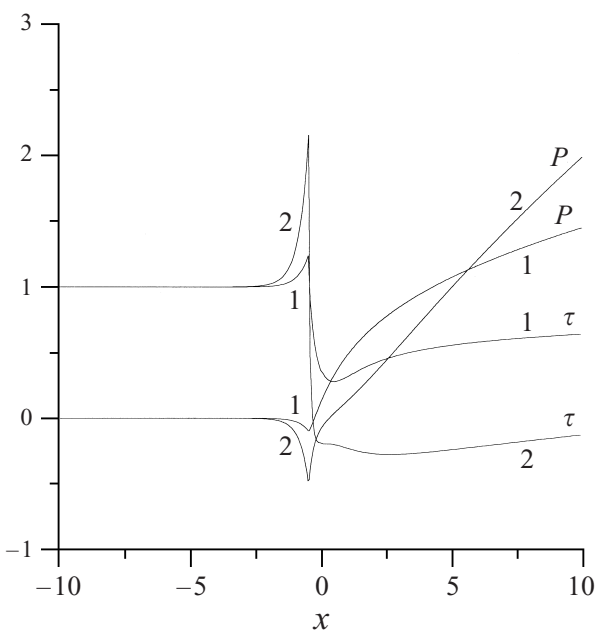

(b)

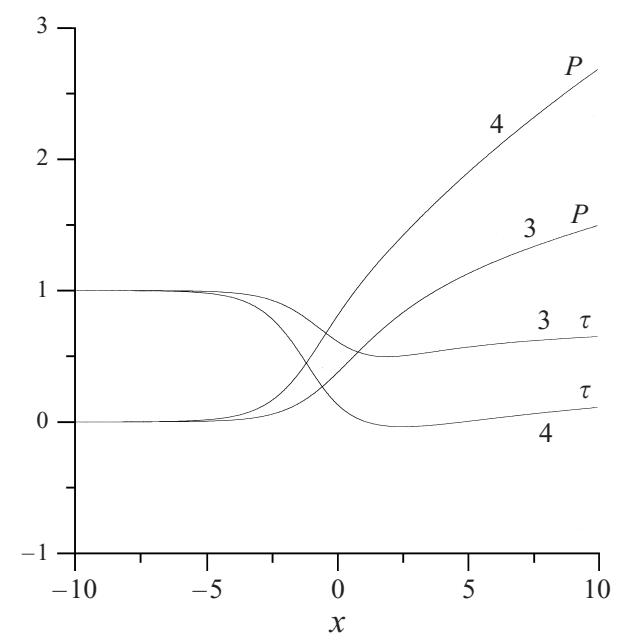

(c)

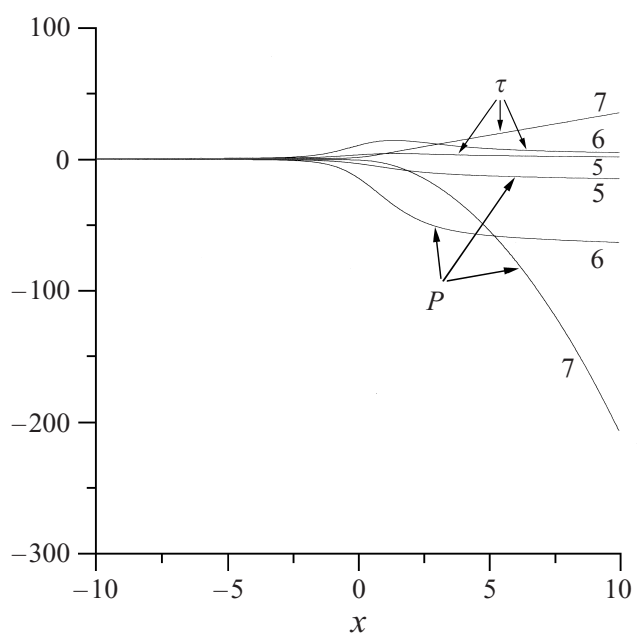

FIGURE 4. $(a-c)$ Viscous wall-layer results computed in seven cases 1-7 (in each the divider shape and wall shape are specified as shown graphically), showing the induced pressures, wall shears and effective viscous displacements, along with the prescribed divider (dvdr) and wall shapes (gsh) for comparison. 
flow turning downstream together with a uniform increase in the gap width, as reflected in the corresponding displacement effects $B$ shown. One case (2) has a larger angle of turning, however, and this alters $B$ sufficiently that wall-layer separation (negative $\tau$ ) is provoked downstream of the start of the turning, accompanied by a relatively rapid rise in the induced wall pressure. The downstream separation occurs despite the increased flow attachment upstream represented by increased $\tau$ values and a strengthened pressure drop. Figure 4(b) in contrast shows the effects of keeping the divider shape and the flow turning angle fixed but varying the wall shape such that the downstream gap width is varied. The increase in the latter width from case 3 to case 4 just produces flow separation again with an enhanced adverse wall-pressure gradient, mainly because the imposed distortion of the wall shape (giving area expansion) is larger everywhere upstream and so $|B|$ is also larger. Figure 4(c) also has the divider shape kept fixed and straight but the three cases shown $(5,6,7)$ all have outer wall shaping that yields a contraction of the area, either with overall flow turning (cases 5, 6) or without (case 7). Each of the three yields a monotonically increasing response in $B$ and hence enhanced shear and monotonically falling pressure at the outer wall throughout the branching, and the increases in these effects from case 5 to 6 to 7 are exactly in line with the associated increases in area contraction.

The results establish that for flow reversal to be avoided or confined the key function $B$ in $(2.2 c-e)$ must be kept at $O(1)$ typically. Even though the central divider shape $f_{1}(x)$ generally provokes upstream acceleration $\left(u_{W}\right.$ positive), suggesting a favourable wall-pressure gradient and increasing wall shear in the wall layer for $x<0$, this can readily be followed by deceleration and then flow reversal after the branch in $x>0$. The latter occurs if there is too large or too abrupt a flow expansion (with $f_{W}<0$ ), the more so as the thicknesses $\left|f_{1}^{-}\right|$and $\left|f_{W}\right|$ are increased. To counter the trend towards flow reversal the wall deviation $f_{W}$ must usually be allowed to start ahead of $x=0$, as the figures above show, but with suitable shaping in order to keep the combined displacement effect $B$ sufficiently smooth and bounded. Similar reasoning applies downstream, where with overall flow turning present $f_{1}^{-}$leads to the slip (3.5), while $f_{W} \sim-h\left(\tilde{\gamma}_{0}+\tilde{\gamma}_{1} x\right)$ say, with $\tilde{\gamma}_{0}, \tilde{\gamma}_{1}$ constants, and so from $(2.2 c)$

$$
B \sim h\left(\gamma_{1}-\tilde{\gamma}_{1}\right) x+h\left(\gamma_{0}-\tilde{\gamma}_{0}\right) \quad \text { as } \quad x \rightarrow \infty .
$$

Only the increase in gap width $\left(f_{W}-f_{1}^{-}\right)$matters here, as might be expected physically. In particular, if the daughter width tends to a constant $D W$ downstream, with $D W=1+R^{-1 / 3} \hat{\beta}$ then the geometry requires $\gamma_{1}=\tilde{\gamma}_{1}, h\left(\tilde{\gamma}_{0}-\gamma_{0}\right)=\hat{\beta}$. That yields an effective area expansion if $\hat{\beta}>O\left(\tilde{\gamma}_{0}>\gamma_{0}\right)$. This expansion can also preserve attached flow, or confined reversal of flow, provided $B$ which is negative in (3.6) remains $O(1)$ then, from the results in figure 4, i.e. provided $\hat{\beta}$ remains $O(1)$. Thus, in principle, a good (attached-flow) design for the branch keeps the area expansion fixed at $O\left(R^{-1 / 3}\right)$, even while allowing further increased turning by means of increased $f_{W}, f_{1}^{-}$.

\subsection{Substantial flow turning}

Substantial turning of the flow, e.g. by $45^{\circ}$, can likewise be achieved without substantial flow separation, in principle at least. The $O(1)$ wall-shape deviation produced then has to extend upstream again, to counteract the increased slip velocity provoked by the $O(1)$ central divider thickness and maintain $|B|$ at $O(1)$. The extension upstream in fact must continue for a distance which is logarithmically large in $R$, from Smith $(1978,1979)$ and from the behaviour of $u_{W}$ as $x \rightarrow-\infty$. In addition, concerning the downstream response in the branched motion, the width $D W$ must remain as 


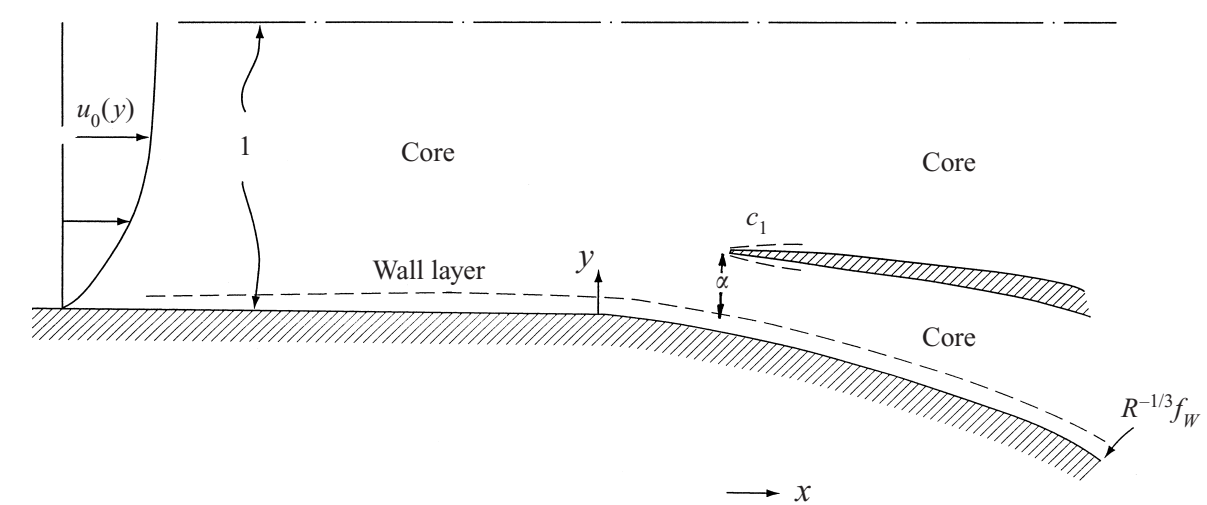

FIGURE 5. Branching for an increasingly small daughter tube, of width $\alpha$, as studied in $\S 4$ : the flow structure.

before, $1+R^{-1 / 3} \hat{\beta}$ with $\hat{\beta}$ order unity, if both area expansion overall and suppressed separation are desired. If the wall shape does not deviate (expand) so much upstream, then the viscous wall-layer motion becomes strongly attached far ahead of the divider, because of the enhanced upstream influence. This strong attachment or contraction in effect may be gained at the expense of the wall layer flow being unable to negotiate smoothly the remainder of the branching, with its eventual area expansion required overall downstream. Conversely, if the wall shape produces too much area expansion upstream of the divider then the viscous wall layer separates far in advance, as in Smith $(1978,1979)$, an equally undesirable design property. So, while the above confirms that within the limitations of the current theory separation-free expansion of area can be achieved/designed even with substantial turnings by the one-to-two branch, the shape design is a rather delicate one.

\section{With a small daughter (near-wall branch)}

Here $N=2$ and the daughter gap widths are $\alpha, 1-\alpha$, with $0<\alpha<1$. There are really three daughters across the whole channel, by symmetry, but we still need consider only half the channel, as sketched in figure 5. There is also special concern for small $\alpha$ values; two new mechanisms then become apparent as the near-wall behaviour proves dominant. Again the mass flux increments are specified: $c_{1}$ in the near-wall daughter and $-c_{1}$ in the middle one, and the relative effects of these and the divider shapes are to be examined.

\subsection{For gap widths of order unity}

If $\alpha$ is of order unity then the core-flow problem $(2.1 a-d)$ is still difficult for an arbitrary curved profile $u_{0}(y)$ when $N=2$, owing to the mixed boundary conditions, but significant progress can be made for the profiles $u_{0}=y$ or 1 . Then $(2.1 a)$ reduces to Laplace's equation and a conformal mapping procedure can be applied, giving the flow solution exactly, for the simplistic case $u_{0} \equiv y$ (see $\S 3.1$ ),

$$
\begin{array}{r}
\tilde{\psi}=\frac{c_{1}}{\pi}\left(\theta_{1}-\theta_{2}\right), \quad \tan \theta_{1,2} \equiv \bar{y} /(\bar{x} \mp 1), \\
\pi \zeta=-\alpha \ln \left(\frac{\bar{\zeta}+1}{\alpha}\right)-(1-\alpha) \ln \left(\frac{\bar{\zeta}-1}{1-\alpha}\right)+\ln 2+\mathrm{i} \pi,
\end{array}
$$



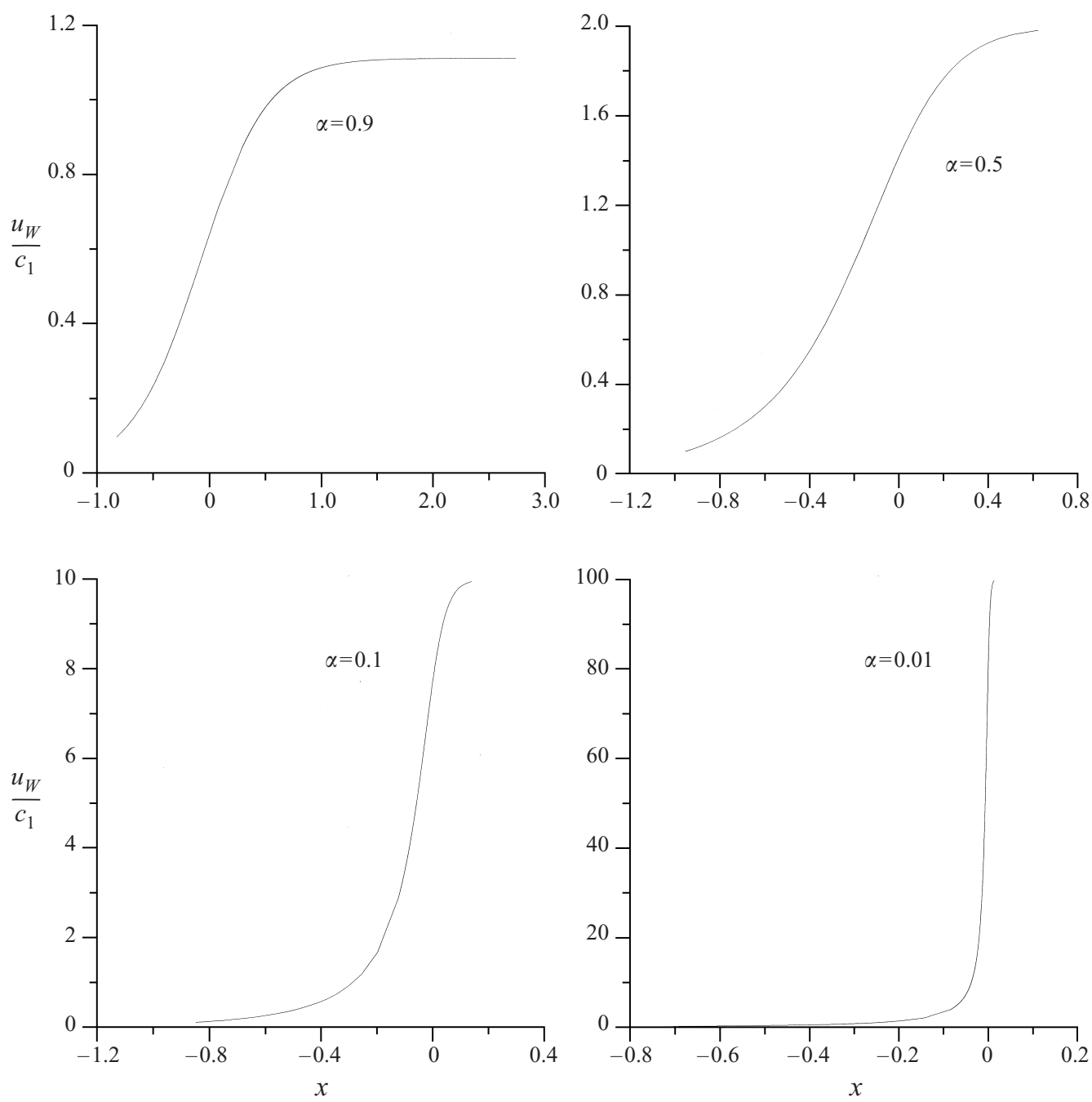

FIGURE 6. Computed slip velocity for various $\alpha$ values from the inviscid core.

where $\zeta, \bar{\zeta}$ denote $x+\mathrm{i} y, \bar{x}+\mathrm{i} \bar{y}$ in turn. Here (4.1c) serves to define $\bar{x}, \bar{y}$. The resulting slip velocity,

$$
u_{W}=-2 c_{1}(\bar{x}+1-2 \alpha)^{-1},
$$

is plotted against $x$ in figure 6 for various $\alpha$ values. Clearly it is positive (negative) if $c_{1}$ is positive (negative). The above is for straight divider shapes, general thickness effects being incorporated near the end of this section, whereas comparisons with results for other $N$ values are presented in the following section.

The solution in (4.1a)-(4.2) takes an interesting two-regional form as $\alpha$ is decreased toward zero (for a near-wall branching), namely

$$
\begin{aligned}
& \tilde{\psi}=c_{1}(1-y)-\frac{c_{1}}{\pi} \tan ^{-1}\left[\frac{\sin (\pi y)}{\exp (\pi x)-\cos (\pi y)}\right], \\
& \tilde{\psi}=c_{1}-\frac{c_{1}}{\pi} \tan ^{-1}(\bar{Y} / \bar{X}),
\end{aligned}
$$




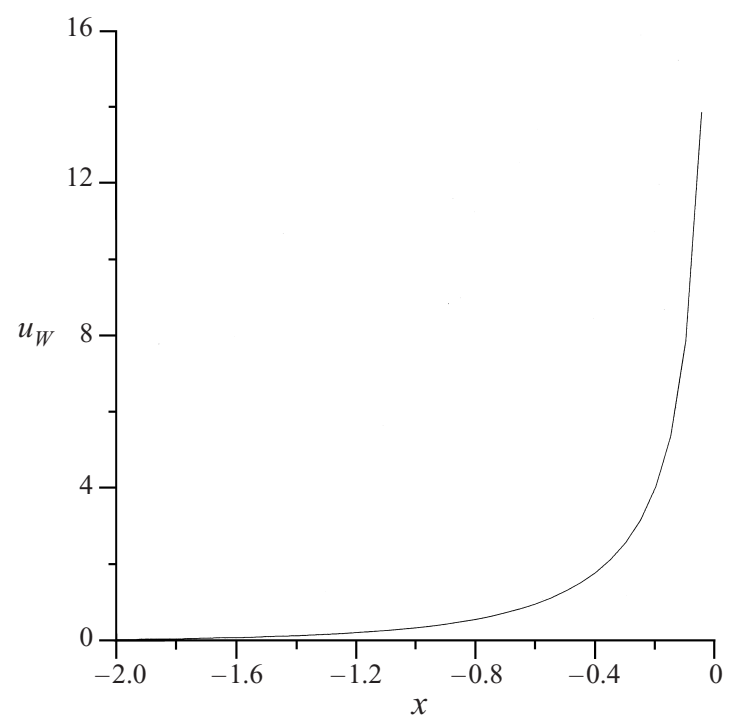

FIGURE 7. The slip velocity from the inviscid solution for small $\alpha$ leading up to the near-wall branch. The subsequent response through the mouth of this branch is described in the text near $(4.5 a, b)$.

to leading orders, with $\bar{x}+1=\alpha \bar{X}, \bar{y}=\alpha \bar{Y}$. Here (4.3a) applies everywhere except in an $O(\alpha)$ region close to the near-wall entrance $x=0$, where $(4.3 b)$ holds with

$$
\pi \zeta=[-\ln (\bar{X}+\mathrm{i} \bar{Y})+(\bar{X}+\mathrm{i} \bar{Y}) / 2+\ln 2-1+\mathrm{i} \pi] \alpha
$$

being of order $\alpha$ (say $\zeta=\alpha(\hat{X}+\mathrm{i} \hat{Y})$ where $x=\alpha \hat{X}, y=\alpha \hat{Y})$. The induced slip accordingly develops two length scales $|x|$, one of $O(1)$ in which $u_{W} \sim c_{1} /(\exp (-\pi x)-1)$ upstream for $x<0$, showing exponential decay far upstream followed by algebraic growth as $x \rightarrow 0-$, and the other near the entrance where $|x|$ is $O(\alpha)$ and $u_{W}=\alpha^{-1} c_{1} /(2+\bar{X})$ is notably large (here $\left.\pi \hat{X}=\ln |\bar{X}|+\bar{X} / 2+\ln 2-1, \bar{X} \sim 1\right)$. The latter $u_{W}$ form decays to match the former as $\hat{X}(\approx \bar{X} / 2 \pi) \rightarrow-\infty$, upstream, whereas downstream as $\hat{X} \rightarrow \infty(\bar{X} \rightarrow-1)$ the slip $u_{W}$ tends to the comparatively large value $\alpha^{-1} c_{1}$ at the end of the $O(\alpha)$ entrance region. This larger slip downstream is consistent with the mass flux increment $c_{1}$ in the small daughter branch. See also figure 6 .

\subsection{Small gap, general input profile}

The small- $\alpha$ core response for a general $u_{0}(y)$ profile then follows. Thus, away from the near-wall mouth, $(2.1 a, c)$ still hold in most of the core (region I) but $(2.1 b, d)$ are replaced by

$$
\begin{gathered}
\tilde{\psi}=0 \quad \text { at } y=1 \quad(\text { all } x), \\
\tilde{\psi}=\left\{\begin{array}{llll}
0 & \text { at } & y=0 & (x<0) \\
c_{1} & \text { at } & y=0 & (x>0) .
\end{array}\right.
\end{gathered}
$$

The mass-flux requirement $\left(c_{1}\right)$ is felt in this outer motion, whereas other details of the near-wall mouth are unseen. The solution of $(2.1 a, c),(4.4)$ can be obtained in series similarly to that in $\S 3$, for plane Poiseuille input for instance, and shows exponential behaviour far upstream but algebraic blow-up in $u_{W}$ as $x \rightarrow 0-$, as demonstrated in figure 7 for the slip velocity obtained from solving in the Poiseuille case.

Close to the mouth, on the other hand, the incident profile is linear to a first 

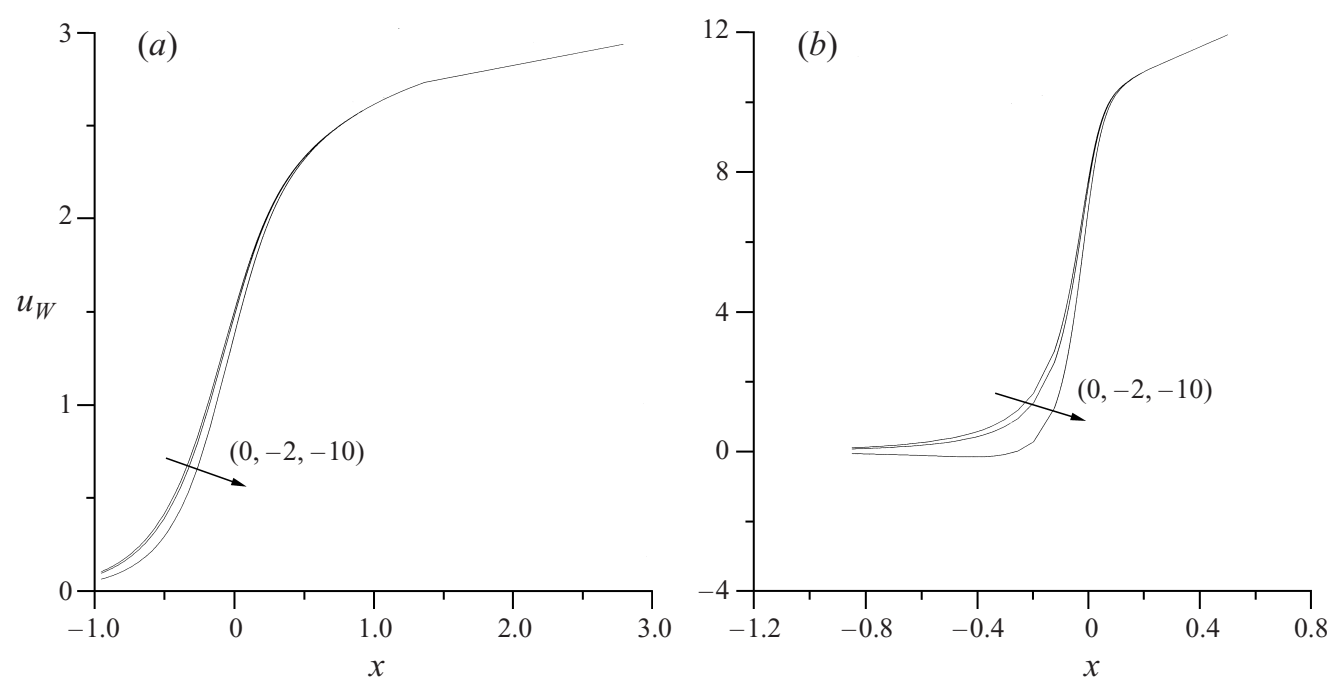

FIGURE 8. Thickness effects for divider shape $\left(f_{1}^{+}, f_{1}^{-}\right)=(H N, H M)(1-\exp (-x)) / u_{0}(\alpha)$ (with parameter values $H M=0,-2,-10$ and $H N$ set at 0.5 ), for $(a) \alpha=0.5,(b) \alpha=0.1$.

approximation and so $(2.1 a)$ becomes Laplace's equation in the coordinates $\hat{X}, \hat{Y}$ (the mouth region II of order $\alpha$ by $\alpha$ ) subject to

$$
\begin{gathered}
\tilde{\psi}=0 \quad \text { along } \quad \hat{Y}=0 \quad \text { for all } \hat{X}, \\
\tilde{\psi}=c_{1} \quad \text { along } \quad \hat{Y}=1 \pm \quad \text { for } \hat{X}>0,
\end{gathered}
$$

from $(2.1 b, d)$, and to matching with the outer solution above. Now the (simple) geometry of the mouth is seen of course. The near-mouth solution, satisfying (4.5), is therefore exactly as in $(4.3 b, c)$, for any input profile $u_{0}(y)$. In particular $u_{W}$ becomes typically large, of order $\alpha^{-1}$, in the mouth region II where $|x|$ is $O(\alpha)$ and inside the small daughter branch.

\subsection{Divider thickness effects}

The addition of thickness effects along the branch at $y=\alpha$ allows arbitrary daughter shapes to be accommodated also. Core solutions for a wide range of different thicknesses $f_{1}^{ \pm}(x)$ at $y=\alpha$, with zero thickness $f_{2}$ at the centreline, were obtained by extending the conformal mapping approach of (4.1) in the case of incident uniform shear and are presented in figure 8 , for two values of $\alpha$. The value of $c_{1}$ is kept at unity. For each $\alpha$ value figure 8 shows the effects on the induced slip velocity of increasing the divider thickness. The indication is that as the gap width $\alpha$ becomes smaller the influence of the daughter mass-flux parameter $c_{1}$ tends to swamp the thickness effects, within bounds, and certainly so near the mouth region.

With such thicknesses present the core-flow structure becomes three-regioned (I-III) as $\alpha$ tends to zero, for the general incident profile $u_{0}(y)$. Region I is as before, in (4.4), as a rule, since the outside shape $f_{1}^{+}$of the daughter channel cannot enter play because of the factor $u_{0}\left(y_{n}\right)(\approx \lambda \alpha)$ multiplying it in $(2.1 b)$; an exception occurs if $\left|f_{1}^{+}\right|$is itself of order $\alpha^{-1}$ (or larger), although this does not alter the flow response near the mouth. Region II, the order $\alpha$ by $\alpha$ mouth region, is likewise as before, in (4.5), leading to the enhanced slip velocity $u_{W} \sim \alpha^{-1} c_{1}$ downstream at large positive $\hat{X}$ just inside the small branch. Region III consists of the slender remainder of the small-branch flow, where $x$ is of order unity but $y$ is small, $0<y<\alpha$. So there the 
governing equation reduces to $\partial^{2} \tilde{\psi} / \partial y^{2}=0$ in effect, yielding $\tilde{\psi}=\left(c_{1}-M(x)\right) y / \alpha$ and hence, to leading order,

$$
u_{W}(x)=\alpha^{-1}\left(c_{1}-M(x)\right),
$$

with $M(x)$ denoting $\lambda \alpha f_{1}^{-}(x)$ from $(2.1 b)$ and usually being considered small. The thin-gap result (4.6) matches with the region II solution at $x=0+$ and with the far downstream asymptote $\alpha^{-1} c_{1}-\lambda f_{1}^{-}(\infty)$ of $u_{W}$ when, say, the inner thickness is constant there. A fourth region also suggests itself, along the outside of the small daughter with $y$ of order $\alpha$ for $x>0$ and similarly for $x<0$, but this is passive. Close agreement with the three-regioned behaviour is evident in the results of figure 8 .

\subsection{The wall layer}

The ensuing viscous wall-layer properties are shown in figure 9. Figure $9(a)$ has increasingly positive values $(1,8,40)$ of $c_{1}$ prescribed, which yield increasingly positive displacements $B$ and hence increased shear and pressure drops along the outer wall. Figure $9(b)$, which is for increasingly negative $c_{1}$ values $(-2,-4)$, shows $B$ becoming more negative and so inducing greater falls in the shear accompanied by rising pressure, with the $c_{1}$ value of -4 leading to a separation near the mouth of the branch.

The viscous flow properties are therefore analogous with those in the previous section except that here the major variation in the slip $u_{W}$ and hence in the key displacement $B$, especially for small $\alpha$, is usually close to the daughter mouth on the $O(\alpha)$ streamwise length scale, and is dominated by the $c_{1}$ mass-flux contribution to $u_{W}$. Aside from extreme conditions, the shapes of the small daughter walls are insignificant locally then, although they can reassert control further downstream, and the characteristic length of upstream influence is lessened compared with that in the previous section. Close to the mouth, positive $c_{1}$ values corresponding to an increase in flux through the near-wall daughter lead to an acceleration of the viscous sublayer, a favourable wall pressure-gradient response and hence increasing wall shear. As $u_{W}$ approaches its nominal local maximum $\alpha^{-1} c_{1}$ just beyond the mouth, the induced wall pressure $P$ tends to $-\frac{1}{2} \alpha^{-2} c_{1}^{2}$ (Smith 1978) (or more generally $-\frac{1}{2} B^{2}$ ), the wall shear $\tau$ decaying like the inverse square root of distance as the wall layer motion becomes very attached typically. This is unless $c_{1}$ is reduced to $O(\alpha)$ : beyond that, the pressure and shear variation is more gradual and wall-shape effects $f_{1}^{-}, f_{W}$ may enter the reckoning, as in (4.6). Negative $c_{1}$ values, in contrast, associated with decreased daughter-channel flux, provoke rapid wall-layer deceleration and flow reversal, usually of a breakaway separation form as $\alpha$ reduces, inducing adverse pressure gradients.

So reduction of separation would point to $c_{1}$ having to be positive. Then, in addition, an improvement of overall flow turning and area expansion seems possible; $f_{1}^{ \pm}, f_{W}$ all being negative produce both turning and expansion even in the absence of $f_{2}^{-}$and the motion then remains separation-free provided that the daughter-gap increment proportional to $\left(f_{1}^{-}-f_{W}\right)$ (cf. the previous section) does not increase too rapidly in $x$ downstream.

\subsection{Comments}

Two mechanisms are important in most of the above flow features. One concerns the mass-flux effect $c_{1}$ through the daughter, which is crucial near the outside wall, including in the viscous wall layer, and is generally far stronger than the wall-shape effect. The other is due to the branching being near the wall ( $\alpha$ small), which reduces the typical distance of the major upstream influence, overwhelming the effects of 

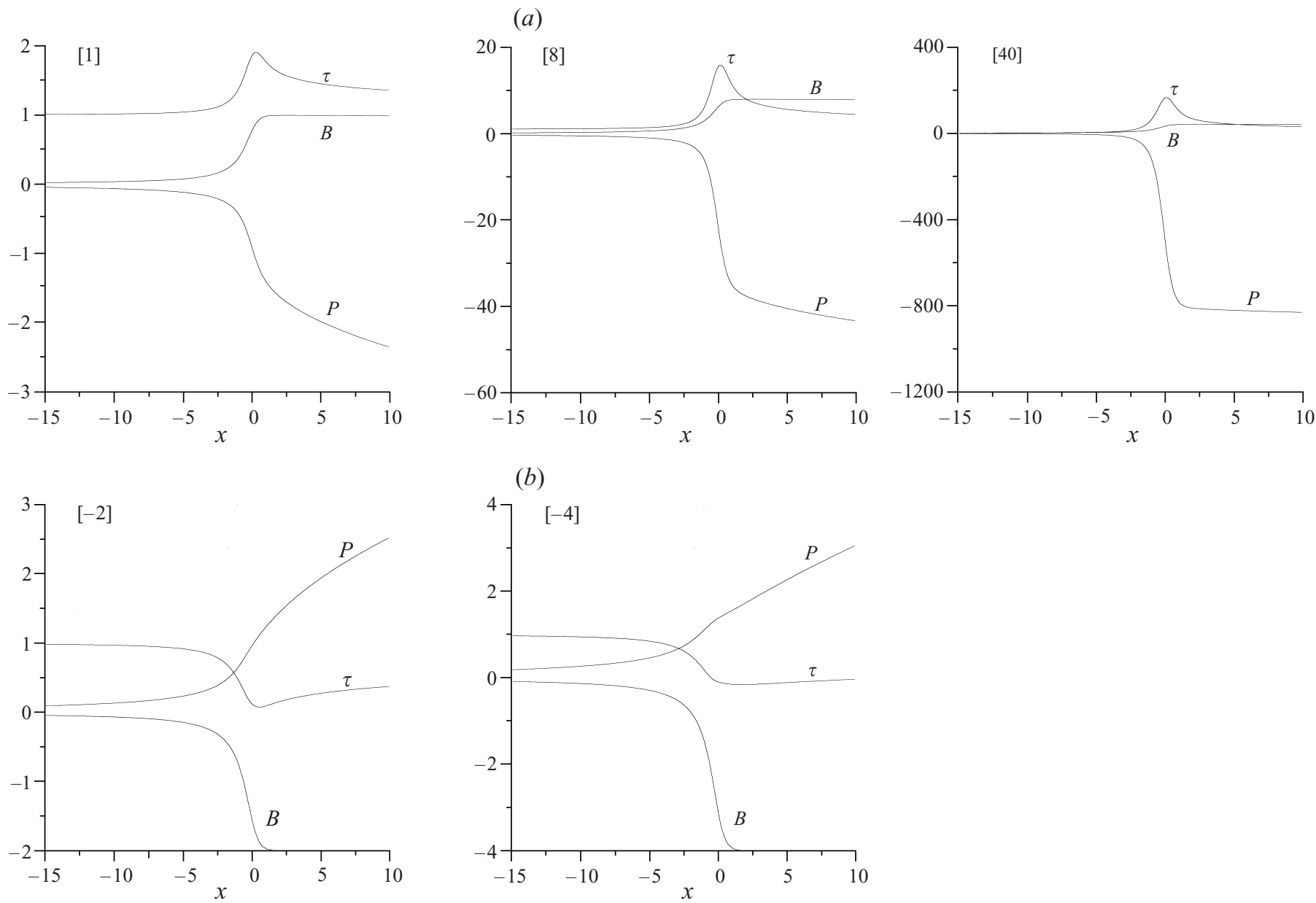


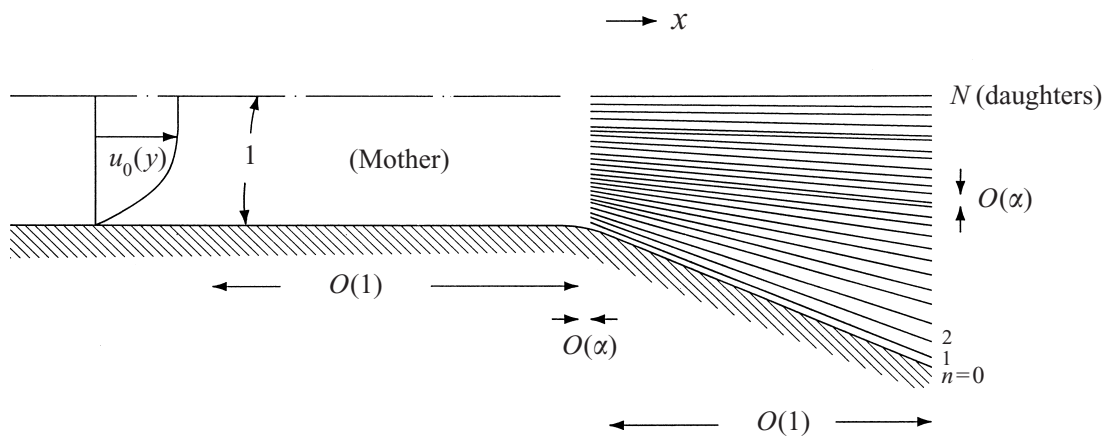

FIGURE 10. Branching with many small daughters: $\S 5$. For general incident profile $u_{0}$, the flow structure with large $N$, small $\alpha$, including the main three streamwise length scales of the mother, mouth and daughter flows and the two lateral length scales.

increased thickness or mass flux unless the latter are extreme, and is allied with the parabolic thin-gap property (as in (4.6)) describing the flow inside the thin daughter channel thereafter. This reduction in upstream influence is tantamount to a discontinuity being present in the flow solution (when viewed on the $|x| \sim 1$ length scale with $y$ of order $\alpha$ for example) between the upstream and downstream solutions, as in the abrupt leading-edge adjustments described by Jones \& Smith (2000), Jones (2000), Bowles \& Smith (2000), Smith (2000b) for airfoil-ground, car-ground and blade-wake interactions in external flows.

We note moreover, concerning the theoretical limitations again, that if $\alpha$ is reduced further to $O\left(R^{-1 / 3}\right)$ the thin-gap flow on the $O(1)$ scale in $x$ becomes viscous-inviscid provided that the mass flux $c_{1}$ is also reduced by the same factor $O\left(R^{-1 / 3}\right)$, whereas if $c_{1}$ is maintained at order one, say, as $\alpha$ is reduced then the near-wall flow soon becomes much more multi-structured, including the abrupt adjustment close to the mouth as indicated above. That abrupt adjustment is in general forced to be nonlinear when $\alpha$ is decreased to the order $R^{-1 / 6} c_{1}^{1 / 2}$; the inviscid nonlinear solution then is still given by $(4.3 b, c)$ however, locally, as described by Jones \& Smith (2000).

The significance of the mass-flux and small-gap properties here leads on to the study of a multiple branch in the following section.

\section{With many small daughters (multiple branching)}

In a multiple branching $\alpha$ is small with $N$ large, such that $\alpha N$ remains $O(1)$; see figure 10. The number of branches $N$ is also assumed to be less than $O\left(R^{1 / 3}\right)$, which is about 10 say, because of the restrictions of the theory. The inviscid core is considered first.

\subsection{Arbitrary number of daughters}

We start by generalizing the mapping technique (Schwarz-Christoffel) from (4.1) to an arbitrary number $N$ of equi-spaced daughters with gap width $\alpha($ here $\alpha N=1)$ and thin walls, for the input profile $u_{0}=y$. As in the previous two sections this $u_{0}$ profile is rather simplistic but it does lead on to subsequent generalization. A suitable map from the branched channel to the upper half $-\bar{\zeta}$ plane is

$$
\zeta=C_{1}+\kappa \pi \mathrm{i}-\kappa \sum_{j=1}^{N} h_{j} \ln \left(\bar{\zeta}-\bar{x}_{j}\right),
$$


where the constant $C_{1}$ is real, for later convenience we use $\kappa \pi=1$ which is the channel half-width, $\bar{x}=\bar{x}_{j}$ are the $N$ mapped downstream ends of the daughters and, for equal spacings, $h_{j}=N^{-1}(=\alpha)$. With $\bar{x}=\bar{x}_{k}^{*}$ denoting the $(N-1)$ mapped leading-edge points, the constraints

$$
\prod_{k=1, k \neq j}^{N}\left(\bar{x}_{j}-\bar{x}_{k}\right)=N \prod_{k=1}^{N-1}\left(\bar{x}_{j}-\bar{x}_{k}^{*}\right)
$$

then apply for $j=1$ to $N$ as the branches are identical. Hence the $\bar{x}_{k}^{*}$ values for $k=1$ to $(N-1)$ are the roots of the polynomial

$$
\sum_{j=1}^{N} \prod_{k=1, k \neq j}^{N}\left(\bar{x}-\bar{x}_{k}\right)
$$

in $\bar{x}$. Further, since all the leading edges $\zeta=\zeta_{k}^{*}(k=1$ to $N=1)$ in the channel must have the same real part, to line up, the $\bar{x}_{j}$ values for $j=2$ to $(N-1)$ are the roots of the polynomial

$$
\sum_{k=1}^{N-1} \frac{(-1)^{N-k}}{\left(\bar{x}_{k}^{* 2}-1\right)} \prod_{j=1, j \neq k}^{N-1} \frac{\left(\bar{x}-\bar{x}_{k}^{*}\right)}{\left(\bar{x}_{j}^{*}-\bar{x}_{k}^{*}\right)}
$$

in $\bar{x}$, with $\bar{x}_{N}, \bar{x}_{1}$ set at \pm 1 respectively. So an iterative scheme can be used, finding $\bar{x}_{k}^{*}$ from the roots of $(5.2 a)$ for given $\bar{x}_{j}$ values, then new $\bar{x}_{j}$ from the roots of $(5.2 b)$ for given (latest) $\bar{x}_{k}^{*}$ values, and so on. Finally, the constant $C_{1}$ is chosen so that the real part of $\zeta_{k}^{*}$, which at iterative convergence is constant over $k$, is identically zero.

The flow solution now follows as

$$
\tilde{\phi}+\mathrm{i} \tilde{\psi}=\pi \kappa \mathrm{i}-\kappa \sum_{j=1}^{N}\left(c_{j}-c_{j-1}\right) \ln \left(\bar{\zeta}-\bar{x}_{j}\right),
$$

with velocity potential $\tilde{\phi}$. The above map can be extended to unequal spacings of the daughters and to arbitrary starting points (leading-edge positions), for instance as in branchings through successive generations, and the flow solution can be extended to include wall thicknesses, in principle. Numerical results are presented in figure 11 for $N=2,4,8$ equal daughters with, across the tube, small alterations $\pm \epsilon$ to the successive mass-flux distributions in each daughter. The figure shows in each case not only the disturbance field $\tilde{\psi}$ but also the complete streamline pattern $\psi_{0}(y)+\epsilon \tilde{\psi}$ for the two values $\epsilon= \pm 0.2$ (when $u_{0}=y$ ), to illustrate the distinct effects of the mass-flux distributions.

\subsection{Many daughters, general input profile}

When the gap width $\alpha$ becomes small, the flow develops an interesting substructure. For increasingly large $N$, the $\bar{x}_{j}$ values approach the distribution $\bar{x}_{j}=$ $-\cos [(j-1) \pi /(N-1)]$, in line with the computational solutions. From the substitution $(j-1)=(N-1) \theta$, the derivative of the map obtained from $(5.1 a)$ then tends to the integral form

$$
\frac{\mathrm{d} \zeta}{\mathrm{d} \bar{\zeta}}=-\kappa \int_{0}^{1} \frac{\mathrm{d} \theta}{(\bar{\zeta}+\cos \pi \theta)},
$$

formally, at almost all positions $\zeta$ outside the daughter channels. The integral here is $\left(\bar{\zeta}^{2}-1\right)^{-1 / 2}$ and so the limit map is

$$
\zeta=C_{2}-\kappa \cosh ^{-1}(\bar{\zeta})
$$


20
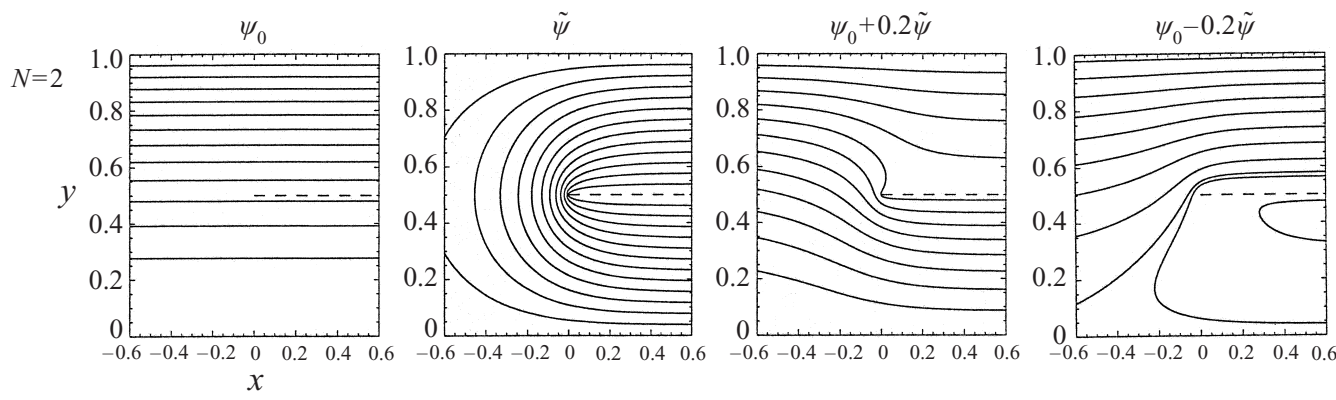
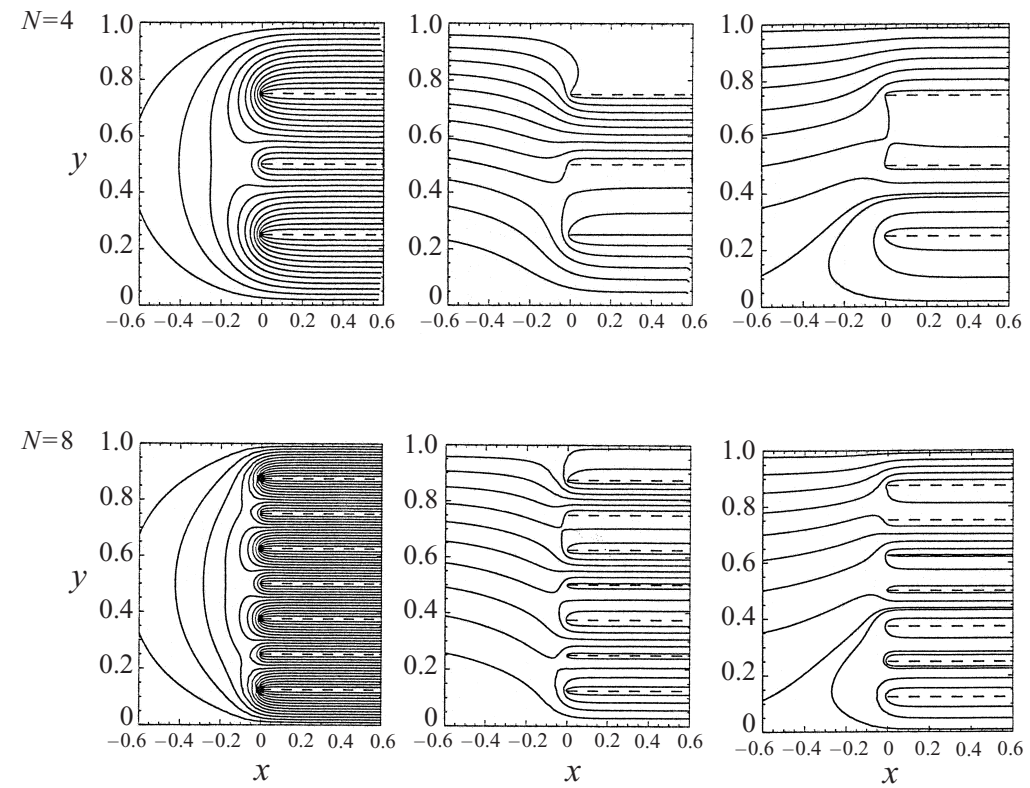

FIGURE 11. Inviscid core results for increasing $N$, decreasing $\alpha$, with incident uniform velocity or uniform shear.

for large $N$, with $C_{2}$ a generally complex constant of integration but to be taken as zero here, along with $\bar{y} \geqslant 0$, so that $\bar{\zeta}=-\cosh (\pi \zeta)$. This map is simply that for the mother channel alone, $0<y<1, x<0$, being transformed to the upper half- $\bar{\zeta}$ plane. See figure 12, which shows the core flow disturbance, the slip velocity and the map for a wide range of values of $N$ (from use of (5.1)-(5.2)) and in which the closeness of the results for values of $N$ above approximately 3 to those for asymptotically large $N$ (obtained from (5.4)) stands out.

The implication is that, in most of the mother flow, the properties on order-unity global scales matter more than the tiny daughter ones, and indeed the above shows the flow structure there to be governed by the total/integrated flux entering the daughter at each point $y$ at the branching station $x=0$. Thus $\tilde{\psi}$ is given by a single Cauchy integral in $\bar{x}, \bar{y}(>0)$, consistent with the limit of $(5.2 c)$, and $u_{W}(x)$ follows as a single Cauchy principal-value integral multiplied by the mapping factor $-\pi \sinh (\pi x)$ (for $x<0)$ almost everywhere in the mother. The flow properties are distinct in or beyond the mouth of any given tiny daughter channel, however, whether near the outer wall or not, as can be seen in the reasoning from (5.1) to (5.3). Whereas the multitude of daughters act to impose a mass-flux distribution spanning the whole channel width, 
Branching tube flows
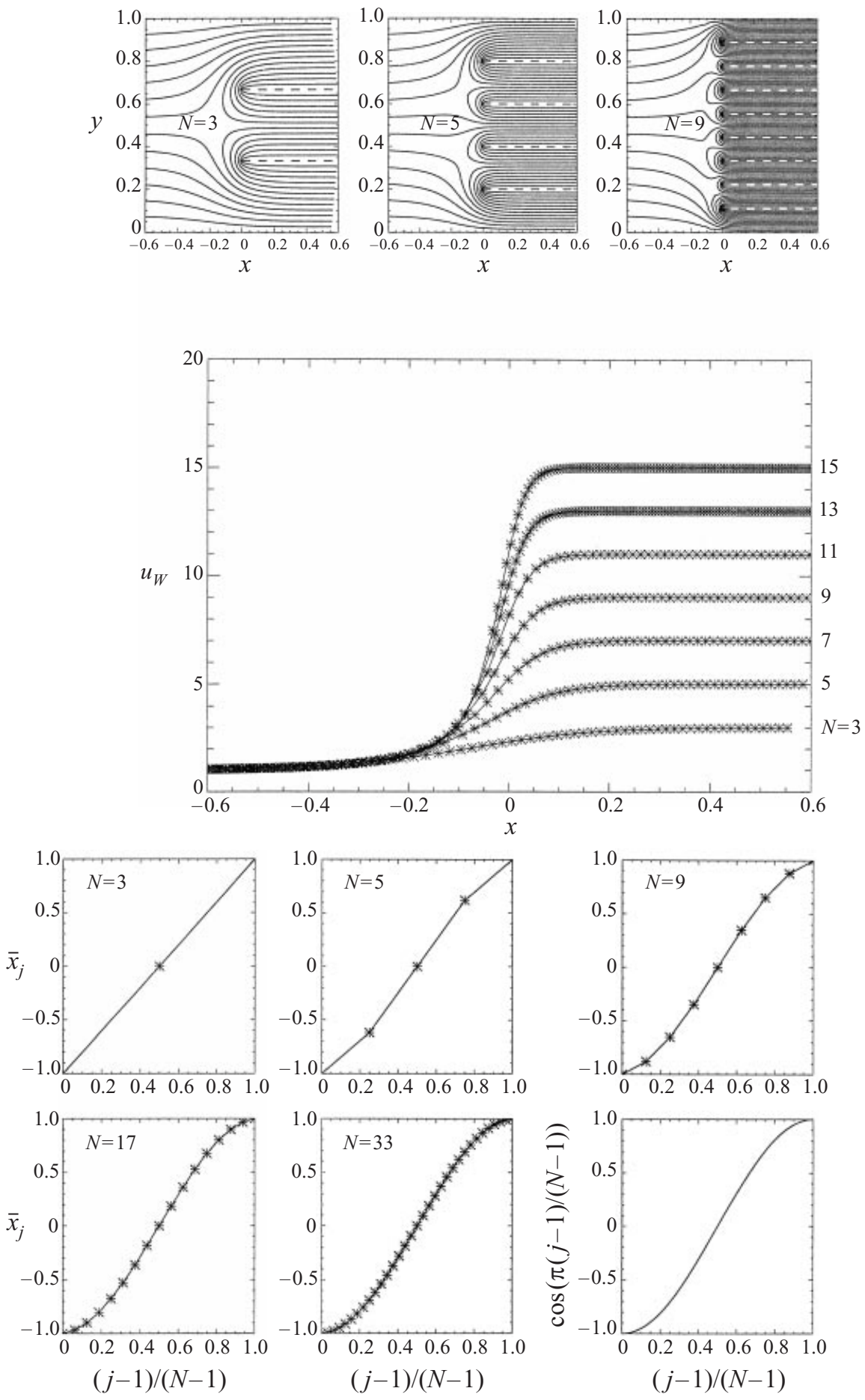

FIGURE 12. Increasing- $N$ and large- $N$ inviscid results and comparisons: the streamlines for successive mass fluxes \pm 1 across the tube, the wall velocities, and the $\bar{x}_{j}$ distribution, compared with the limit case which is shown last. 
thus controlling the mother flow almost everywhere, in contrast each individual daughter flow develops (once beyond the mouth) as if on its own, independently of the rest of the flow field and subject only to its own mass-flux conservation. These features of mother and daughter apply also to unequally spaced small daughters.

The same new features of global-mother and local-daughter flow apply for a general incident profile $u_{0}(y)$ and arbitrary daughter spacings when $\alpha$ is small and $N$ large. Most of the mother flow now is governed by $(2.1 a, c, d)$ still, restricted to negative $x$, subject to the mass-flux requirement

$$
\tilde{\psi}=C(y) \text { at } x=0-
$$

Here $C(y)$ denotes the limiting mass-flux value at each $y$, defined if necessary by an average $\left(c_{n}+c_{n+1}\right) / 2$ at the daughter mouth $y=\left(y_{n}+y_{n+1}\right) / 2$. Following the comments in the introduction concerning flow control, the flux function $C(y)$ is taken as specified first, an example being presented in figure 13(a). This particular flux function is also smooth in $y$, given that individual discontinuities at individual daughters do not affect the global solution. For the case of plane Poiseuille flow $u_{0}=y-y^{2} / 2$ the solution of $(2.1 a, c, d)$ with (5.5) is obtainable similarly to that in $\S 3$ and is shown in figure $13(a)$, in terms of the induced slip velocity at the outer wall upstream of the multiple branch. Second, and again following the comments on flow control, figure 13(b,c) shows the upstream flow solution for the alternative of specified entry pressures $\tilde{p}(y)$ at $x=0$, from solving the pressure equation just below $(2.1 d)$ for all negative $x$, along with zero $\tilde{p}$ upstream and at the walls. The induced velocity $\tilde{u}(y)$ at $x=0$ is also included in the figure, and here we refer forward to $\S \S 6$ and 7 on three-dimensionality and on pressure conditions. The similarity between the upstream results for flux control and pressure control in figure 13 is notable. Downstream almost every small daughter flow in positive $x$ is governed by small-gap balances, with a uniform entry profile in effect as each $y_{n+1}-y_{n}$ gap is small, of order $\alpha$. Consequently there

$$
\tilde{\psi}=\left[\left(y-y_{n}\right)\left(c_{n+1}-u_{0} f_{n+1}^{-}\right)-\left(y-y_{n+1}\right)\left(c_{n}-u_{0} f_{n}^{+}\right)\right] /\left(y_{n+1}-y_{n}\right)
$$

for general daughter wall shapes $f_{n+1}^{-}(x), f_{n}^{+}(x)$, with $y_{n}<y<y_{n+1}$ and $u_{0}$ nominally evaluated at $y_{n}$.

\subsection{Upstream influence and the wall layer}

Some significant upstream influence, due to the overall branching into multiple small daughters, thus occurs on an $O(1)$ length scale in $x<0$, and this contrasts with the case of a single small daughter in the previous section. The present upstream influence also forces the viscous wall layer motion to be nonlinear, via the slip $u_{W}(x)$, at $O(1)$ distances ahead of the branching. On the other hand, within small $O(\alpha)$ distances of the branching, properties of the local individual branches must affect the core near the outer wall, where those branches appear as a semi-infinite stack of dividers in the presence of an incident linear (near-wall) velocity profile. The local core solution, in the $O(\alpha)$ by $O(\alpha)$ mouth region, is therefore exactly as in (5.1) and (5.2) with $N \rightarrow \infty$ but $\alpha$ replaced by unity, to a leading approximation (and with $\kappa$ large), for the example of equi-spaced daughters with thin walls: see figure 12 .

The outer mouth region yields a local change in the slip $u_{W}(x)$ in particular, near the branching. The main question then, especially for the viscous sublayer, is whether or not there is a change in the order of magnitude or character of $u_{W}$. The answer depends first on the local form of the flux function $C(y)$ in (5.5). A broad case has $C$ tending to a non-zero constant $\tilde{C}_{0}$ as $y \rightarrow 0+$, corresponding to significant flux passing into the near-wall daughters, and then $\tilde{\psi}$ near the origin is $2 \tilde{C}_{0}\left(\pi-\tan ^{-1}(y / x)\right) \pi^{-1}$, 

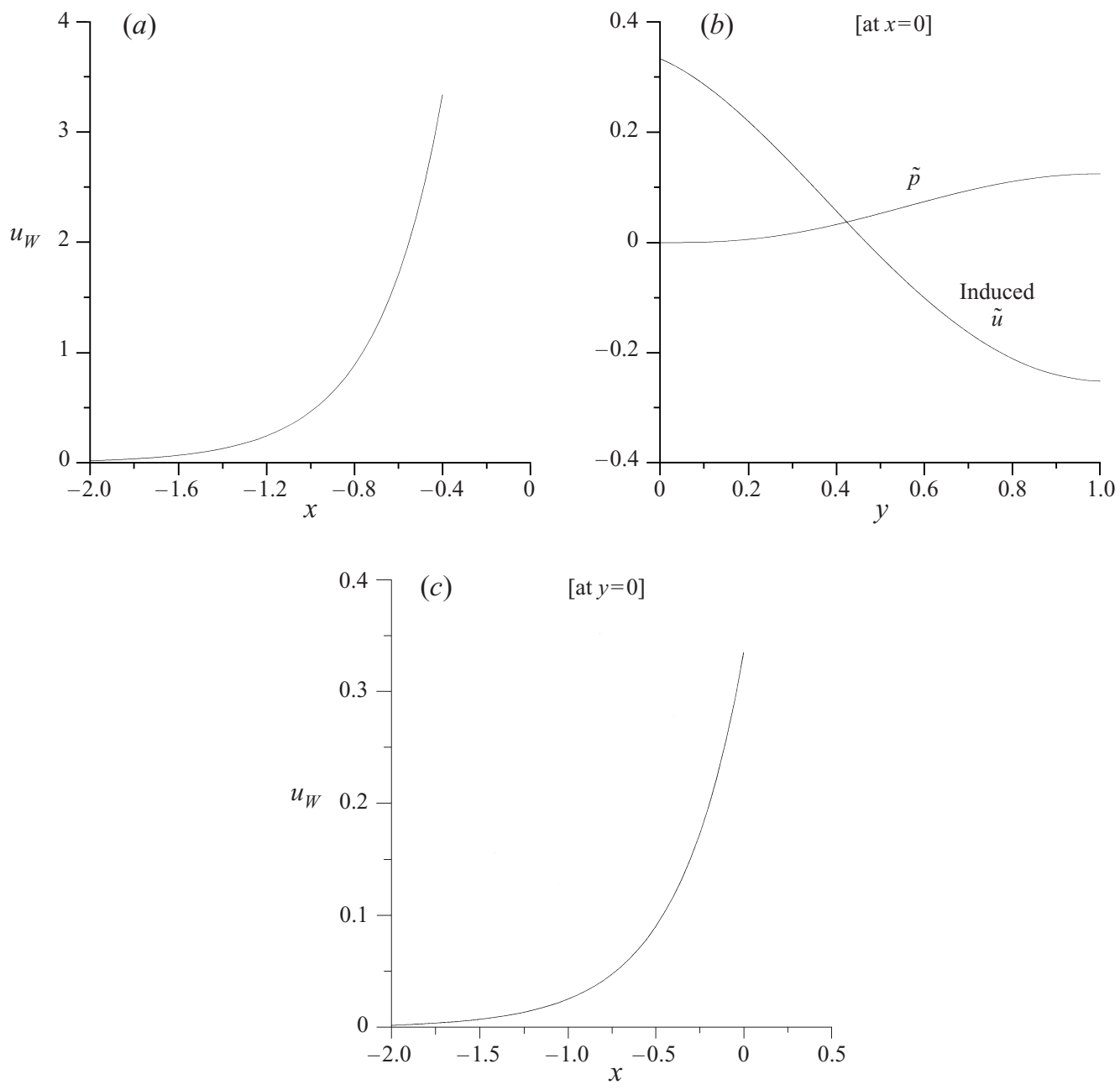

FIGURE 13. (a) For general input profile $u_{0}$ and large $N$, the mother-flow solution for $u_{W}$ in $x<0$ is shown for the specified mass-flux distribution $C(y)=\sin \pi y$ at the branching station. $(b, c)$ As $(a)$ but with a specified pressure distribution (as shown) at the branching station.

from $(2.1 a, d),(5.5)$, leaving $u_{W}(x) \approx 2 \tilde{C}_{0} /(\pi|x|)$ at small negative $x$. Hence there is indeed a substantial change in the order of magnitude of $u_{W}$ in this case, to the size $O\left(\alpha^{-1}\right)$ as the $O(\alpha)$ mouth region is entered, analogous with the behaviour of $u_{W}$ in $\S 4$. (If $C$ tends to zero like $y$, yielding less near-wall flux, as in the example of figure $13(a)$, then $u_{W}$ is logarithmic at small negative $x$, yielding a lesser change.) Accordingly, the viscous wall-layer response is also analogous to that in $\S 4$, being dominated by its development within the short $O(\alpha)$ mouth scale where $\hat{X} \sim 1$. The precise $u_{W}(\hat{X})$ variation there depends on all the nearby $c_{n}$ fluxes (see figure 12). Downstream, as $\hat{X}$ increases $u_{W}$ approaches the constant value $\alpha_{1}^{-1} c_{1}$, to preserve the mass flux $c_{1}$ in the daughter channel nearest the wall. This indicates acceleration of the viscous sublayer taking place throughout the $\hat{X}$ mouth scale if both $\tilde{C}_{0}$ and $c_{1}$ are positive, the trend of the wall-layer behaviour being similar to that in $\S 4$. Exceptional cases might occur, however, involving both rapid acceleration and rapid deceleration in the mouth region, although none have been encountered as yet. 
5.4. Comments

Much more turning and area expansion can be achieved, without flow reversal, in this multi-branching configuration compared with the previous ones, at least on the present streamwise length scales and provided the mass flux into the near-wall daughters is sufficiently positive overall (to avoid the acceleration-deceleration noted above). This is because, while the expansion of area closest to the wall must still be limited to prevent downstream separation in the viscous wall layer as before, the majority of the many daughters across the channel can each expand (and turn) at will, without producing flow reversal. Blasius-like attached boundary layers persist on each such daughter wall and these boundary layers are stronger on the branches near the middle since $u_{0}$ is larger there. The slip velocities from $\partial \tilde{\psi} / \partial y$ remain higher-order effects in each daughter away from the outer wall, and, as remarked earlier, each of these multiple daughter flows acts independently after a small entry distance of order $\alpha$. This inference is confined to within the current theoretical scales of course, and other local multi-branching features can arise if $\alpha$ is reduced to the size $R^{-1 / 6} \tilde{C}_{0}^{1 / 2}$ generally (cf. §4).

\section{Three-dimensional branching, with two or many daughters}

Mechanisms similar to those in $\S \$ 2-5$ also act in a multiple three-dimensional branch, starting at $x=0$ say. A representative configuration is drawn in figure 14 . As the lower parts of the figure indicate, the present work applies in principle for any cross-sectional shapes of daughters, subject as before to the gaps having more cross-sectional area than the dividers (the Smith $2000 a$ study removes this restriction). There is however at least one important possible distinction from the planar case.

\subsection{The core flow and wall layer}

The core flow, as a perturbation of an incident motion $u=u_{0}(y, z)$, is considered for convenience (Smith 1976a, 1977a) in terms of its pressure equation,

$$
\nabla^{2} \tilde{p}=2\left(u_{0 y} \tilde{p}_{y}+u_{0 z} \tilde{z}_{z}\right) / u_{0}
$$

with $\nabla^{2}$ now signifying $\left(\partial_{x}^{2}+\partial_{y}^{2}+\partial_{z}^{2}\right)$. This is subject to the conditions of tangential flow along the separation-free dividers $\left(y=y_{n}(x, z) \pm\right)$, zero pressure disturbance upstream, and pressure matching at the outer wall defined by $g_{0}(x, y, z)=0$ say, so that

$$
\begin{gathered}
\tilde{p}_{t}=-u_{0}^{2} \partial_{x}^{2} f_{n}^{ \pm} \quad \text { at } \quad y=y_{n} \pm, \\
\tilde{p} \rightarrow 0 \text { far upstream, } \\
\tilde{p} \rightarrow 0 \text { at the outer wall }\left(g_{0}=0\right) .
\end{gathered}
$$

The coordinates are as in figure 14, and in particular $t$ denotes the inward normal distance from a wall. The dividers are specified with $y_{n}(z)$ and thicknesses $f_{n}(x, z)$ in $(6.1 b)$, analogous with the planar case of $\S \S 2-5$, so that here they have $y-y_{n}$ small and proportional to $f_{n}$, although this can be generalized if $y=y_{n}$ is multivalued. Mass-flux and/or pressure conditions in each daughter tube are assumed as previously. Similarly the tangential-flow constraint represented by $(6.1 d)$, which is analogous with the condition $(2.1 d)$ in planar flows, implies that $\tilde{p}$ is $O\left(t^{3}\right)$ and the scaled radial velocity $\tilde{v}$ is $O(t)$ near the outer wall, at which $u_{0}$ tends linearly to zero. The solution of (6.1) is to give the slip velocity $u_{W}(x, z)$ driving the viscous sublayer at the outer wall, whereas the boundary layers on each internal divider again 

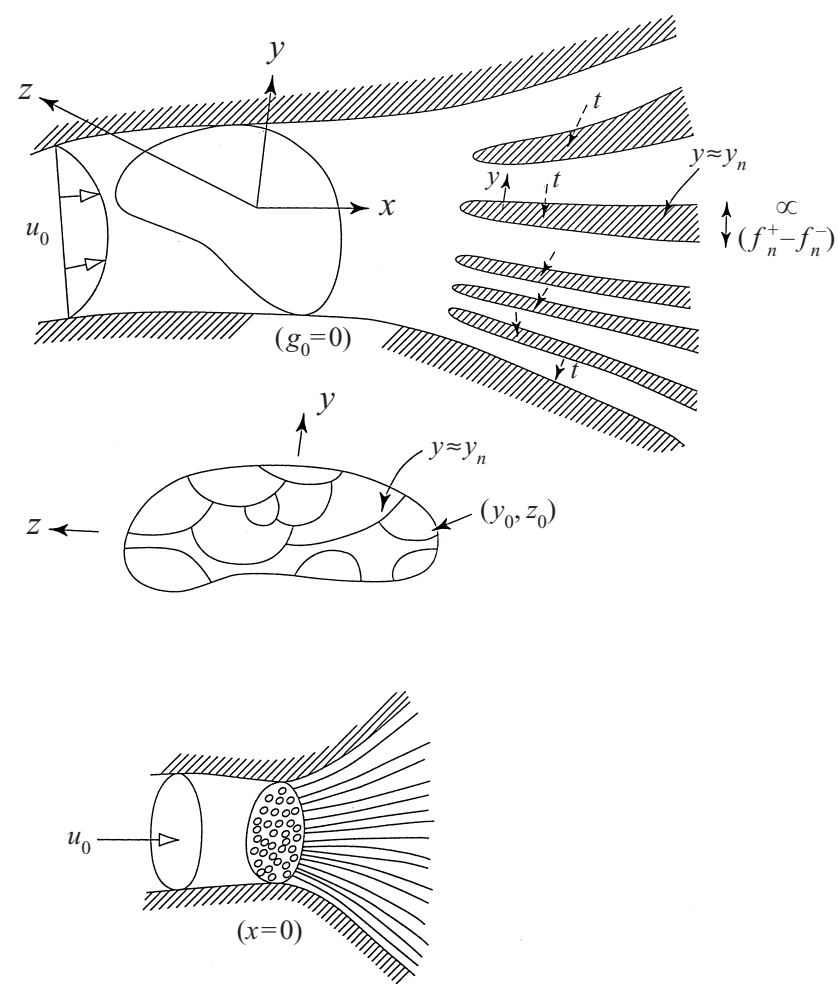

FIGURE 14. Three-dimensional branching, as considered in $\S 6$. Sketch of the flow structure.

remain attached and Blasius-like, as in Smith $(1976 a, 1977 a)$. The slip velocity $u_{W}$ is determined by $2 \lambda \partial^{2} u_{W} / \partial x^{2}=\left(\partial^{3} \tilde{p} / \partial t^{3}\right)$ (at $\left.g_{0}=0\right)$ where $\lambda(z)$ is now the given, positive, scaled wall shear of the incident profile $u_{0}(y, z)$.

The distinction from the two-dimensional case is that here a significant and delicate flow response can occur near the intersection between a divider and the outer wall, similar to a wing-body junction, if geometrical effects rather than mass-flux or pressure effects dominate. The local flow behaviour then depends on the divider and wall shapes near the junction $y=y_{0}(x), z=z_{0}(x)$ generally, but we suppose primarily that the intersection is normal in the cross-plane. Then the governing equation $(6.1 a)$ locally has $\partial_{x x}$ suppressed, to a first approximation, subject to $(6.1 b, d)$ but with $u_{0}$ replaced by $\lambda t$. If the coordinates are rotated such that the divider occupies $y=a, z>b$ locally (with $a, b$ dependent on $x$ ), then $\tilde{p}$ nearby satisfies $\left(\partial_{y}^{2}+\partial_{z}^{2}\right) \tilde{p}=2 \tilde{p}_{z} /(z-b)$ along with the conditions $\tilde{p}=0$ at $z=b$ and $\tilde{p}_{y}=-\tilde{\lambda}^{2}(z-b)^{2} \tilde{f}^{\prime \prime}(x)$, where $\tilde{\lambda}, \tilde{f}$ denote $\lambda, f_{n}$ evaluated at $z=b$. The outer wall is given by $z=b, y>a$, above the junction, and $t$ is identified with $z-b$. With those conditions the local solution must involve a logarithmic term (Walton \& Smith), such that $\tilde{p} \sim \tilde{r}^{3} \ln \tilde{r}$ where $\tilde{r}$ is the distance $\left[(y-a)^{2}+(z-b)^{2}\right]^{1 / 2}$, and hence the slip velocity locally grows as

$$
u_{W} \sim \ln (y-a) \quad \text { as } \quad y \rightarrow a+
$$

along the outer wall. The largest effect is thus at the junction. This same conclusion is reached, albeit with algebraic rather than logarithmic growth, if the thickness $f_{n}$ becomes large, rather than of order unity locally. Again, if the intersection angle is $\beta$ rather than $\pi / 2$, then $u_{W}$ is found to be $O(1)$ and proportional to $1 / \cos \beta$, instead 
of (6.2), but here we shall keep to the normal intersection. In view of the logarithmic response above, all the divider wall thicknesses and mass fluxes or pressure increments are taken to be smaller than in the planar case, by a logarithmic factor, so that they are of order $R^{-1 / 3} / \ln R$. Then both the core flow and most of the viscous wall layer are linear, while nonlinear behaviour is focused in the viscous corner motion. This focusing mechanism, which is special to the three-dimensional case, is equivalent to that in Walton \& Smith (1997); the corresponding corner vortex-flow problem is still unsolved. Below we assume a linear viscous wall layer as it represents most of the induced near-wall flow.

\subsection{One-to-two branchings}

The branchings of most interest currently are those of one-to-two branching (large daughters) and one-to-many (small daughters). For the former, $N=1$, the effects of a thin divider placed symmetrically in a straight circular pipe are studied by Smith (1977a), Bennett (1987, wherein he notes an error in the 1977a paper which was pointed out to him by his supervisor F.T.S. and which leads to the present flow structure) and recently by Blyth \& Mestel (1999). By contrast, the present work considers a thicker divider, with $f_{1}^{ \pm}$non-zero but equal, splitting a circular mother pipe, $r=1$ in cylindrical polar coordinates, into two equal semi-circular daughters which are not necessarily straight. The core exhibits some upstream influence for such a configuration: see figure 15 below, including secondary flow. In the viscous wall layer therefore the linearized three-dimensional boundary-layer equations hold,

$$
\begin{gathered}
U_{x}+V_{Y}+W_{z}=0, \\
\lambda Y U_{x}+\lambda V=-P_{x}(x, z)+U_{Y Y}, \\
\lambda Y W_{x}=-P_{z}(x, z)+W_{Y Y},
\end{gathered}
$$

where $\lambda$ is constant in this case and $u=R^{-1 / 3}(\lambda Y+U / \ln R)$. The presence of the logarithmic term may be an extra restriction on the practical usefulness of the theory, of course, added to the limitations discussed in the introduction. Equation $(6.3 a-c)$ forms the three-dimensional counterpart of $(2.2 a)$ but linearized as expected. The boundary conditions require no slip at the outer wall, given by $Y=f_{W}(x, z) /(\ln R)$, and matching with the core solution, so that

$$
\begin{gathered}
U=V=W=0 \quad \text { at } \quad Y=0, \\
U \rightarrow \lambda B(x, z) \quad \text { as } \quad Y \rightarrow \infty, \quad B \equiv \lambda^{-1} u_{W}(x, z)+f_{W}(x, z), \\
W \rightarrow 0 \quad \text { as } \quad Y \rightarrow \infty,
\end{gathered}
$$

together with the vanishing of $(U, V, W, P)$ upstream as $x \rightarrow-\infty$, and periodicity around the outer wall. The viscous problem (6.3) is virtually identical in essence to Smith's (1976b), the only difference being in the details of the forcing $B$ in $(6.3 e$ ). Hence there is extra upstream influence associated with the three-dimensional case, via the viscous-inviscid mechanism described in the last-named paper.

The main features of the symmetric one-to-two branching flow are thus well known. The theory can cope only with linear effects, because of the nominally small $1 / \ln R$ factor above, which in turn is due to a geometrical effect, and so separation cannot be accommodated; the only major questions arise in the nonlinear corner vortices. This applies even far downstream where $(6.1 a-d)$ yield an analogue of the planar flow result (3.6), but the corner vortices control the restrictions on bending and area expansion for separation-free motion. Again, the arguments above crucially assume 
(a)
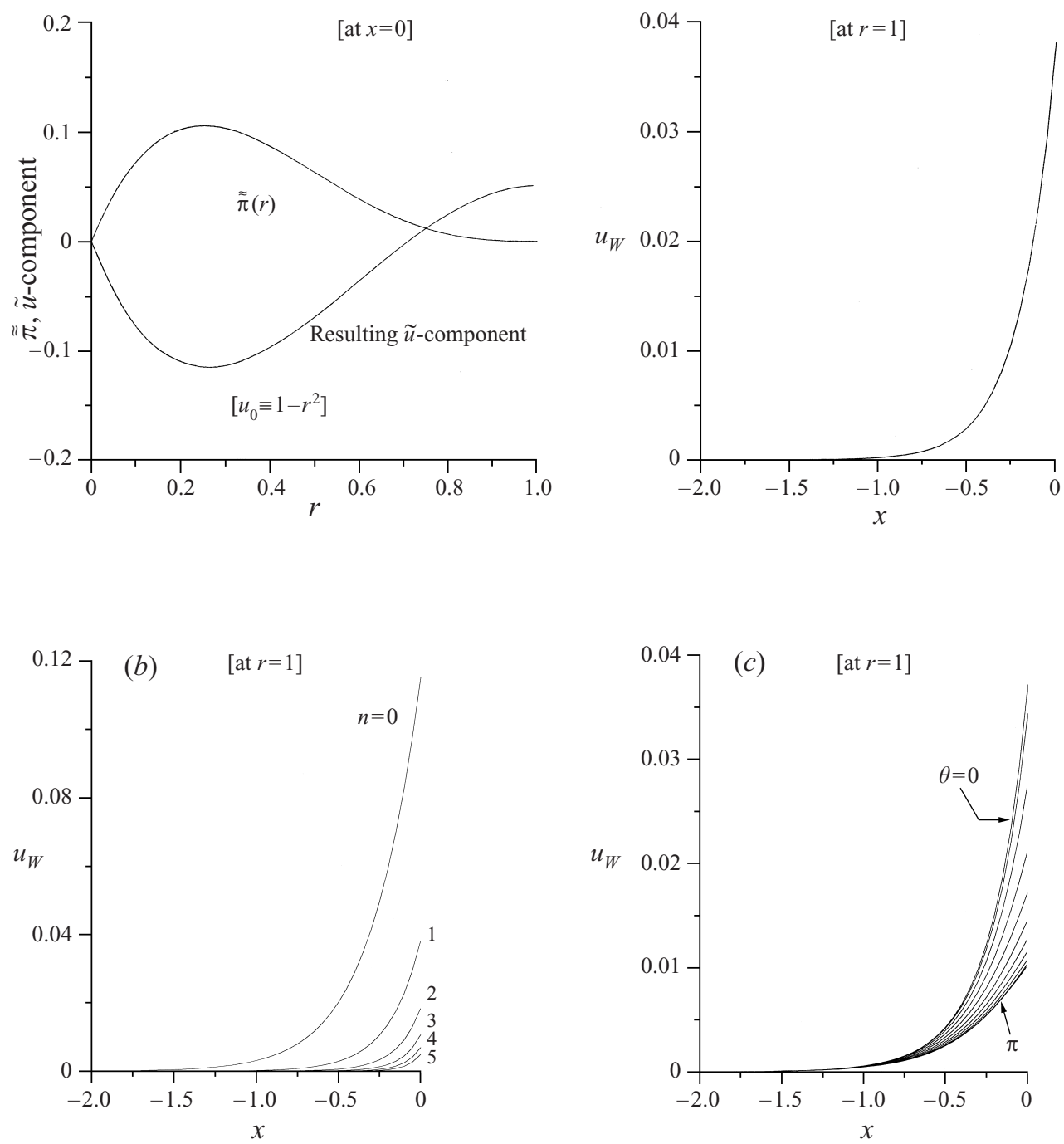

FIGURE 15. (a) For one-to-many branchings: computed results for (on the left) the induced velocity at the branching station and (on the right) the slip velocity upstream, given a single-mode $(\sin \theta)$ pressure distribution as shown at the multi-branching station. (b) As (a) but giving the computed slip velocity $u_{W}$ for modes $\sin (n \theta)$ imposed at the multiple branching. $(c)$ As $(a)$ but computed slip velocity $u_{W}$ for an imposed pressure which is distributed over one quarter of the circular cross-section and zero elsewhere at the branching station.

equal mass flux and pressure distributions in the two daughter tubes, for symmetry, whereas an example of unequal distributions is given below, leading to non-symmetry. The one-to-many case, in contrast, is controlled almost invariably by the daughter pressures or fluxes, and we address this next.

\subsection{One-to-many branchings}

For one-to-many branching with numerous small daughters, $N \gg 1$, the broad argument of $\S 5$ still holds in the sense that the mother flow is controlled by $(6.1 a, c, d)$, 
but now

$$
\tilde{p}=\tilde{\pi}(y, z) \quad \text { at } x=0 .
$$

The right-hand side is the daughter-inlet function defined pointwise by the pressure entering each individual daughter, analogously to $\S 5$. It determines the mother flow throughout $x<0$, and it applies for a general incident profile $u_{0}(y, z)$.

Solutions obtained from mode decomposition are presented in figure $15(a-c)$, for representative $\tilde{\pi}$ distributions which are smooth and zero at the outer wall; the solutions are for fully developed incident flow $u_{0}=1-r^{2}$ and in one case include the induced $\tilde{u}$ distribution at the branching station. The individual daughter flows act separately again, controlled by thin-tube equations, except in the $O(\alpha)$ scaled mouth region(s). There the strongest change in upstream influence is generated, as far as the slip velocity and hence the viscous wall-layer displacement and resulting flow are concerned. This is analogous to the planar case in $\S 5$. Indeed, reasoning exactly as in the planar case points to much increased turning and area expansion, free of separation, because of the incident flow strength for all the many middle daughters, allied with suppression of the geometrical effects relative to the overall pressure distribution. Nonlinear wall layer flow could actually be incorporated here.

The examples from (6.4) shown in figure $15(a, b)$ confirm the existence of nonsymmetric flow for symmetrical geometry occurring due to non-symmetry in the downstream flow conditions. Also, with the surgical procedures of $\S 1$ in mind, we show (through double summation) the influence of altering the daughter pressures within a patch of the multiple branching cross-section, in figure $15(c)$. The case in figure $15(a)$ has $\tilde{\pi}=\tilde{\pi}(r) \sin \theta$ in (6.4), with the function $\tilde{\pi}$ being as shown in the figure, corresponding to increased pressure throughout the upper half of the multiple daughters and decreased pressure in the lower half. The solution of $(6.1 a, c, d),(6.4)$, is then a summation of modes, each with $\tilde{p} \propto \exp \left(\beta_{1 m} x\right) \sin \theta$ multiplied by a function of $r$. The positive eigenvalues $\beta_{1 m}$ are determined similarly to those in $\S \S 3-5$ and the constants of proportionality are found by applying (6.4). The mother flow solution shows deceleration in most of the upper half of the tube upstream as might be expected, with fluid being drawn (pressurized) broadly into the lower half. Close to the outer wall however a compensation takes place, yielding acceleration (deceleration) in the upper (lower) half of the mother tube. The induced velocity profile across the daughter mouths ties in with those trends. In figure $15(b) \tilde{\pi}$ is taken as $\tilde{\pi}(r) \sin n \theta$, with $n$ varying from zero to 5 , and the mother flow solution is derived as for the case $n=1$ above. The decay of the extent of upstream influence with increasing $n$ is clear. Figure $15(c)$ on the other hand has $\tilde{\pi}$ equal to $\tilde{\pi}(r) \cos 2 \theta$ for the patch $|\theta|<\pi / 4$ and zero otherwise. In this case a Fourier series in $\theta$ combined with mode summations of the form used in figures $15(a, b)$ gives the upstream flow solution. The trends suggested by the previous cases are partly present for the patch of figure $15(c)$ but now the zero- $n$ axisymmetric component dominates upstream, as the induced slip velocities demonstrate at various values of $\theta$ for example.

\section{Further comments}

\subsection{Roles of geometry and mass fluxes}

This research has considered the roles of both the geometrical (divider and outer wall) shapes and the mass fluxes of the daughter tube flows at a one-to-few or one-to-many branching, especially for $(a)$ two large daughters, $(b)$ one small daughter/side branch and $(c)$ multiple small daughters. 
Concerning geometry first, in the present context the inviscid core flow is affected mostly by the middle divider shapes and little by the outer wall shape, whereas the viscous wall-layer motion is influenced equally by both, directly or indirectly through the effective displacement $B$. If the geometry alone is important then the middle branches tend to produce an attached trend in the motion at the outer wall, especially upstream in order for the flow to negotiate the branching downstream, whereas those wall shapes that correspond to an overall turning or expansion of the typical cross-sectional tube area tend to produce a separated trend. The balance of these two opposing trends determines the critical expansion ratio $\beta$.

Concerning mass fluxes second, the theory shows that these can actually be more crucial, provoking substantial differences not only between the properties in different daughters but also in the entire mother-tube flow upstream. The increased significance of the daughters' mass fluxes is particularly strong for one-to-many or for side branches. (The role of pressure drops is considered further in $\S 7.3$ below.)

\subsection{Separation-free flow}

The overall flow turning and expansion at a branch themselves give rise to some distinct features, in the multi-branching flows. These seem best considered in terms of the three main configurations $(a)-(c)$ of the study.

The work in $\S 3$ confirms the approximate maximum value of 1.2 for the area expansion ratio $\beta$, to avoid separation in a one-to-two branching $(a)$, in terms of the Reynolds numbers of interest. The solution trends agree with those shown by Hademenos \& Massoud for example. On the other hand, the work indicates that with suitably designed careful shaping of the wall the turning can be enlarged to include typical angles of order unity, rather than the small angles associated with most prescribed wall shapes.

Section 4 shows that, for a side branch $(b)$, the major upstream adjustment occurs instead near the mouth of the small daughter, accompanied by a corresponding downstream adjustment locally but with a major downstream response also taking place on a longer length scale well within the daughter.

The one-to-many branching $(c)$, in $\S 5$, exhibits a similar tendency except that there is, in addition, an upstream adjustment of the entire mother flow and it is controlled totally by the effective mass-flux or pressure distribution of the multiple small daughter system across the tube. The findings hold for arbitrary shapes of these small-daughter flows, as well as arbitrary mass fluxes and arbitrary incident mother-flow profiles $u_{0}$, and moreover the reasoning extends to three-dimensional branches as in $\S 6$. The one-to-many case also suggests that the expansion ratio $\beta$ can be made much greater than 1.2 in the presence of multi-branching, without inducing flow separation and without the careful wall-shape designing needed in the one-to-two case above.

\subsection{On further research}

Design of improved surgical strategies for treating arteriovenous malformations and of improved surgical grafts, among other practical considerations, may be aided only indirectly by features such as those above, involving the many physical mechanisms that operate in branchings, as already indicated briefly in this section and in more detail in $\S \S 3-6$. Further research is certainly required for directly useful applications, for example basic research on nonlinear core flows, for stronger mass-flux variations in the daughter tubes, and on increased turnings for the cases $(b),(c)$. The numerous limitations on the present theory highlighted in the introduction need to be made 
fewer, although the extra limitation on the multiple-daughter case $(c)$, that $N$ should remain small relative to $R^{1 / 3}$ in order for the core response to remain inviscid, is not necessarily severe in practice since the $N$-large results work well for $N$ above about 3 (see figure 12), while $R^{1 / 3}$ is 10 typically. Nevertheless viscous effects are likely to become accentuated almost everywhere in the daughters if there are more than about 6-8 daughters at the branching.

The relation between prescribed-pressure and prescribed-flux daughter flow solutions is quite straightforward for most of the thin daughters in (c), from application of mass and momentum conservation on the present shorter length scale, in keeping with the argument in the introduction on pressure and flux control over longer scales. An exception concerns the prescribing of end-pressure constraints in the outer daughters where the input velocity $u_{0}$ is zero and there is possible interaction between, and upstream influence from, the viscous pressure drop of approximately $-\frac{1}{2} B^{2}$ and the end pressure. Longer viscous scales for the (nonlinear) daughter flows clearly need much study, in two and three dimensions, partly for calculations on pressure control, partly in view of the AVM application above and partly in view of the relatively abrupt pressure or mass-flux adjustment imposed on the $O(1)$ length scale by the longer scale solution, as inferred from Jones \& Smith's (2000) work and described earlier. Study is required likewise for thinner side- or multi-branching daughters; other three-dimensional features, for small side branches for example; and unsteady and/or flexible-wall motions. The interaction between any side-graft flow, branching at one station but re-joining the main core flow downstream, and the core flow itself, is also of interest in terms of the long-scale upstream influence from the end-pressure equality required at the downstream junction (Jones \& Smith 2000).

The authors thank Mr N. D. Kitchen, for observations, comments and questions which led to this research, and the referees and editor for helpful comments. Support for M. A. J. from ESPRC is acknowledged gratefully.

\section{REFERENCES}

BATES, S. 1978 On the effects of junctions, bifurcations, heating and expansions on tube flows. $\mathrm{PhD}$ thesis, University of London.

BennetT, J. 1987 Theoretical properties of three-dimensional interactive boundary layers. PhD thesis, University of London.

Blyth, M. G. \& Mestel, A. J. 1999 Steady flow in a dividing pipe. J. Fluid Mech. 401, 339-364.

Bowles, R. G. A. \& SMith, F. T. 2000 Lifting multi-blade flows with interaction. J. Fluid Mech. 415, 203-226.

Borgas, M. S. \& Pedley, T. J. 199, Non-uniqueness and bifurcation in annular and planar channel flows. J. Fluid Mech. 214, 229-250.

Brotherton-Ratcliffe, R. V. 1987 PhD thesis, University of London.

Dennis, S. C. R. \& Smith, F. T. 1980 Steady flow through a channel with a symmetrical constriction in the form of a step. Proc. R. Soc. Lond. A 372, 393-414.

Fisher, M. \& Fieman, S. 1990 Geometric factors of the bifurcation in carotid atherogenesis. Stroke 21, 267-271.

Gao, E., Young, W. L., Pile-Spellman, J., Joshi, S., Duong, H., Stieg, P. E. \& Ma, Q. 1997 Cerebral arteriovenous malformation feeding artery aneurysms; a theoretical model of intravascular pressure changes after treatment. Neurosurgery 41, 1345-1358.

Hademenos, G. J. \& Massoud, T. F. 1997 Biophysical mechanisms of stroke. Stroke 28, 2067-2077.

Hademenos, G. J., Massoud, T. F. \& ViÑuela, F. 1996 A biomathematical model of intracranial arteriovenous malformations based on electrical network analysis: theory and hemodynamics. Neurosurgery 38, 1005-1015.

Handa, T., Negoro, M., Miyachi, S. \& Sugita, K. 1993 Evaluation of pressure changes in feeding 
arteries during embolization of intracerebral arteriovenous malformations. J. Neurosurg. 79, 383-389.

Jones, M. A. 2000 Mechanisms in wing-in-ground-effect dynamics. PhD thesis, University of London. Jones, M. A. \& Smith, F. T. 2000 Fluid motion for car undertrays in ground effect. Phil. Trans. R. Soc. Lond. A (theme issue), accepted for publication.

Lei, M., Kleinstreuer, C. \& Archie, J. P. 1997 Hemodynamic simulations and computer-aided designs of graft-artery junctions. J. Biomech. Engng 119, 343-348.

Lighthill, M. J. 1972 Physiological fluid dynamics: a survey. J. Fluid Mech. 52, 475-497.

Luo, X. Y. \& Pedley, T. J. 1996 A numerical simulation of unsteady flow in a two-dimensional collapsible channel. J. Fluid Mech. 314, 191-225.

Luo, X. Y. \& Pedley, T. J. 1998 The effects of wall inertia on flow in a two-dimensional collapsible channel. J. Fluid Mech. 363, 253-280.

Miyasaka, Y., Yada, K., Kurata, A., Tokiwa, K., Irikura, K., TanaKa, R., Ohwada, T. \& KitAHARA, T. 1993 Correlation between intravascular pressure and risk of hemorrhage due to arteriovenous malformations. Surg. Neurol. 39, 370-373.

Motomiya, M. \& Karino, T. 1984 Flow patterns in the human carotid artery bifurcation. Stroke $15,50-55$.

RoAch, M. R., Scott, S. \& Ferguson, G. G. 1972 The hemodynamic importance of the geometry of bifurcations in the circle of Willis (glass model studies). Stroke 3, 255-267.

RothmaYer, A. P. \& Smith, F. T. 1998 Incompressible triple-deck theory. In Handbook of Fluid Dynamics, Chaps. 23-25. CRC.

Sмiтн, F. T. $1976 a$ On entry-flow effects in bifurcating, blocked or constricted tubes. J. Fluid Mech. 78, 709-736.

SмIтн, F. T. $1976 b$ Pipeflows distorted by non-symmetric indentation or branching. Mathematika 23, 62-83.

Smith, F. T. 1977 a Steady motion through a branching tube. Proc. R. Soc. Lond. A 355, 167-187.

Sмiтh, F. T., $1977 b$ Upstream interactions in channel flows. J. Fluid Mech. 79, 631-655.

Sмiтh, F. T. 1978 Flow through symmetrically constricted tubes. J. Inst. Maths Applics. 21, 145-156.

Sмiтh, F. T. 1979 The separating of flow through pipes of general cross-section. Mathematika 26, $187-210$

Smith, F. T. 2000a Multiple branching of tube flow: large flow rates and dual motions. In preparation.

Smith, F. T. $2000 b$ On physical mechanisms in two- and three-dimensional separations. Phil. Trans. R. Soc. Lond. A (theme issue), in press.

Smith, F. T. \& DUCK, P. W. 1980 On the severe non-symmetric constriction, curving or cornering of channel flows. J. Fluid Mech. 90, 727-753.

Sobey, I. J. 1980 On flow through furrowed channels. J. Fluid Mech. 96, 1-26.

Tillett, J. P. K. 1968 On the laminar flow in a free jet of liquid at high Reynolds numbers. J. Fluid Mech. 32, 273-292.

Walton, A. G. \& Smith, F. T. 1997 Concerning three-dimensional flow past a tall building on flat ground. Q. J. Mech. Appl. Maths 50, 77-128.

Young, W. L., Kader, A., Pile-Spellman, J., Ornstein, E. \& Stein, B. M. 1996 Arteriovenous malformation drawing vein physiology and determinants of transnidal pressure gradients. Neurosurgery 35, 389-396. 